Article

\title{
Revitalizing Fertility of Nutrient-Deficient Virgin Sandy Soil Using Leguminous Biocompost Boosts Phaseolus vulgaris Performance
}

\author{
Mahmoud A. Abdelfattah ${ }^{1,2, *} \mathbb{C}$, Mostafa M. Rady ${ }^{3, *} \mathbb{C}$, Hussein E. E. Belal ${ }^{3}$, Eman E. Belal ${ }^{1}$, \\ Rahmah Al-Qthanin ${ }^{4,5}$, Hatim M. Al-Yasi ${ }^{6}$ and Esmat F. Ali ${ }^{6}(\mathbb{D}$ \\ 1 Soils and Water Science Department, Faculty of Agriculture, Fayoum University, Fayoum 63514, Egypt; \\ eeb00@fayoum.edu.eg \\ 2 Food and Agriculture Organization of the United Nations (FAO), Cairo 11668, Egypt \\ 3 Botany Department, Faculty of Agriculture, Fayoum University, Fayoum 63514, Egypt; hes00@fayoum.edu.eg \\ 4 Prince Sultan Bin-Abdul-Aziz Center for Environment and Tourism Studies and Researches, King Khalid \\ University, P.O. Box 960, Abha 61421, Saudi Arabia; alqthanin-r@hotmail.com \\ 5 School of Biological Sciences, King Khalid University, P.O. Box 960, Abha 61421, Saudi Arabia \\ 6 Department of Biology, College of Science, Taif University, P.O. Box 11099, Taif 21944, Saudi Arabia; \\ h.alyasi@tu.edu.sa (H.M.A.-Y.); a.esmat@tu.edu.sa (E.F.A.) \\ * Correspondence: maa06@fayoum.edu.eg (M.A.A.); mmr02@fayoum.edu.eg (M.M.R.); \\ Tel.: +20-84-010-923-920-38 (M.M.R.)
}

Citation: Abdelfattah, M.A.; Rady, M.M.; Belal, H.E.E.; Belal, E.E.;

Al-Qthanin, R.; Al-Yasi, H.M.; Ali, E.F.

Revitalizing Fertility of

Nutrient-Deficient Virgin Sandy Soil

Using Leguminous Biocompost

Boosts Phaseolus vulgaris Performance.

Plants 2021, 10, 1637. https://

doi.org/10.3390/plants10081637

Academic Editors: Riccardo Primi,

Mercedes Martín Pedrosa and

George Lazarovits

Received: 16 June 2021

Accepted: 6 August 2021

Published: 10 August 2021

Publisher's Note: MDPI stays neutral with regard to jurisdictional claims in published maps and institutional affiliations.

Copyright: (c) 2021 by the authors. Licensee MDPI, Basel, Switzerland. This article is an open access article distributed under the terms and conditions of the Creative Commons Attribution (CC BY) license (https:// creativecommons.org/licenses/by/ $4.0 /)$.

\begin{abstract}
During the 2019 and 2020 seasons, nutrient-deficient virgin sandy soil was examined along with the investigation of the response of Phaseolus vulgaris plants to soil application with biocompost in integration with chemical fertilizers applied to soil and plants. Four treatments $(100 \%$ of the recommended NPK fertilizer dose (control), 75\% NPK applied to soil + 25\% foliar spray, $75 \%$ NPK applied to soil $+25 \%$ foliar spray + leguminous compost $\left(C_{L}\right)$, and $75 \%$ NPK applied to soil $+25 \%$ foliar spray $+C_{L}$ containing Bacillus subtilis (biocompost; $C_{L} B$ )) were applied in a randomized complete block design. The $75 \%$ NPK applied to soil $+25 \%$ foliar spray $+\mathrm{C}_{\mathrm{L}} \mathrm{B}$ was the best treatment, which exceeded other treatments in improving soil fertility and plant performance. It noticeably improved soil physicochemical properties, including available nutrients, activities of various soil enzymes (cellulase, invertase, urease, and catalase), soil cation exchange capacity, organic carbon content, and $\mathrm{pH}$, as well as plant growth and productivity, and plant physiobiochemistry, including nutrients and water contents, and various antioxidant activities. The results of the 2020 season significantly outperformed those of the 2019 season, indicating the positive effects of biofertilizers as a strategy to combine soil supplementation with NPK fertilizers and allocate a portion of NPK fertilizers for foliar spraying of plants in nutrient-deficient sandy soils.
\end{abstract}

Keywords: virgin soils; bio-organic and chemical fertilizer; soil properties; Phaseolus vulgaris

\section{Introduction}

Sandy soils are characterized by low nutrient content and water holding capacity, which leads to the frequent application of nutrients and water to meet crop requirements and improve soil quality [1-5]. Virgin sandy soils, which were not used for cultivation before [6], are found in nearly all regions of Egypt, from a few hundred square kilometers to more than a hundred thousand kilometers, covering most of Egypt's total area [7-11].

Soil can generally be enriched with nutrients by adding them in solid form or dissolved in water, and the plant can also be nourished directly with nutrients through foliar application. Historically, soil application is the most common fertilization practice, but it depends on several factors including soil and plant characteristics and the physiological state, as well as weather conditions [12]. Generally, the availability of most nutrients is 
limited in soils with higher calcium carbonate content [13], which are common throughout Egypt. However, foliar application improves the uptake and the efficiency of these nutrients [14,15]. Hence, to alleviate micro- and macronutrient deficiencies in sandy soils, foliar fertilization is increasingly adopted [16]. Foliar fertilization results in rapid nutrient absorption and avoidance of several environmental factors such as antagonism, leaching, and deposition of elements [17]. Hence, it can avoid some of the problems of challenges associated with soil application of nutrients in sandy soils. [17,18]. The foliar application does not substitute for soil application, but supplements it $[19,20]$. However, fertilization of major nutrients, especially NPK, is more effective via soil application, whereas secondary nutrients, e.g., calcium, magnesium, and sulfur, as well as micronutrients, e.g., zinc, iron, manganese, copper, molybdenum, and boron, proved to be more effective via foliar application [19].

Chemical fertilizers are essential for plant nutrition, but they can cause environmental pollution [21], particularly nitrogen, which leads to an increase of $\mathrm{NO}_{3}{ }^{-}$ions in the soil [20]; phosphorous may also cause soil contamination with $\mathrm{Cd}^{2+}$, which is readily absorbed and translocated into different parts of the plant [22]. As one of the basic organic fertilizers, leguminous compost can be utilized to reduce the quantities of chemical fertilizers applied $[23,24]$. The use of leguminous compost has multiple benefits including sanitation, reduced mass and bulk, and a lower $\mathrm{C} / \mathrm{N}$ ratio [21]. Besides, it has the potential to maintain the fertility of soils in agricultural systems $[25,26]$. Legume plants can contribute as much as $50-250 \mathrm{~kg} /$ ha of nitrogen $[27,28]$, and their litter can be utilized as a highly valuable compost (organic fertilizer). In contrast, traditional organic materials, e.g., crop residues, animal manure, etc., cannot alone improve soil fertility, as they are usually not available in sufficient quantities and require intensive labor [29].

Continuous use of inorganic fertilizers negatively affects soil fertility as it eventually leads to reduced crop yields. Moreover, its cost is much higher compared with organic fertilizers, which impacts the overall profits of agricultural production. Hence, it is of prime importance to appropriately combine inorganic with organic fertilizers [23] to overcome the environmental impacts and maximize the overall income. The objective of the present study is to evaluate the effectiveness of chemical and organic fertilizers (leguminous biocompost) in improving the fertility of nutrient-deficient sandy soil and its reflection on the growth and yield of Phaseolus vulgaris using a foliar and/or soil addition. We hypothesized that the combined application of soil and foliar chemical fertilizers with a bio-organic fertilizer would improve the fertility of the investigated sandy soil along with the growth and production of Phaseolus vulgaris plants.

\section{Materials and Methods}

\subsection{Experimental Preparation and Setup}

Two field trials were conducted over two consecutive summer seasons (20 February 2019 and 25 February 2020). Each summer season was preceded by the fall season (the first of September 2018 and 2019), which was planted with Egyptian clover (Trifolium alexandrinum) crop mixed with the soil when plants were 10 weeks old. Then, the soil was irrigated and left until the main planting seasons (February 2019 and 2020).

Next to the Agriculture College (Fayoum University) trials station $\left(29^{\circ} 17^{\prime} 06^{\prime \prime} \mathrm{N}\right.$ and $30^{\circ} 54^{\prime} 55^{\prime \prime} \mathrm{E}$ ), an area of sandy soil (not cultivated before) with an area of $600 \mathrm{~m}^{2}$ was employed for this study. This area was characterized by moderate weather throughout the experimental period during the two growing seasons. Average daily temperatures were $24.5 \pm 2.5^{\circ} \mathrm{C}$, mean relative humidity was $64.6 \pm 6.4 \%$, and light/dark averaged throughout the day $12 / 12 \mathrm{~h}$.

Bronco cultivar of Phaseolus vulgaris (L.) was obtained from the Horticultural Research Institute (Egyptian Center for Agricultural Research). A sterile solution of sodium hypochlorite at a concentration of $1 \%$ was prepared to be used to sterilize the surface of the seeds for $5 \mathrm{~min}$, then the seeds were cleaned with distilled water. Then, the seeds were prepared for planting after air drying for $1 \mathrm{~h}$. 
Before sowing and after harvesting the crop in the 2019 and 2020 seasons, the top $0-20 \mathrm{~cm}$ of the soil was sampled $(n=5)$ and analyzed for soil chemical and physical properties, applying the methods detailed in Page et al. [30] and Klute and Dirksen [31].

The soil area specified for this study was divided into 40 experimental units of $10.5 \mathrm{~m}^{2}$ each, excluding the area of the main boundaries and irrigation canals $\left(180 \mathrm{~m}^{2}\right)$. Each unit consists of five rows of $3 \mathrm{~m}$ in length, and each row consists of 30 hills each planted with three seeds. After full emergence, seedlings were thinned to one hill ${ }^{-1}$, counting 150 plants $10.5 \mathrm{~m}^{-2}$. The recommended doses of NPK fertilizers (e.g., ammonium sulfate $(20.5 \% \mathrm{~N})$, Ca-superphosphate $\left(15.5 \% \mathrm{P}_{2} \mathrm{O}_{5}\right)$, and $\mathrm{K}$-sulfate $\left.\left(48 \% \mathrm{~K}_{2} \mathrm{O}\right)\right)$ were added to the soil at a rate of $380 \mathrm{~kg} \mathrm{~N}, 380 \mathrm{~kg} \mathrm{P}_{2} \mathrm{O}_{5}$, and $190 \mathrm{~kg} \mathrm{~K}_{2} \mathrm{O} \mathrm{ha}^{-1}$. During preparation, the soil was supplemented with a half-dose of $\mathrm{N}$ with a full dose of $\mathrm{P}$ and $\mathrm{K}$. Thirty days after sowing (DAS), the soil was supplemented with another half-dose of N. These NPK amounts were applied at $100 \%$ of the recommended doses as a control treatment. The second treatment was $75 \%$ of the recommended NPK doses $\left(0.30,0.30\right.$, and $0.15 \mathrm{~kg}$ per $10.5 \mathrm{~m}^{2}$, respectively) applied to the soil, $+25 \%\left(0.10,0.10\right.$, and $0.05 \mathrm{~kg} 10.5 \mathrm{~m}^{-2}$, respectively) applied as three foliar sprays (each spraying with a third of the 25\%); 15, 30, and 45 days after sowing. Foliar sprays of NPK were performed with a hand atomizer, and the spray solution amount (to runoff) was sprayed, and Tween 20 (a few drops) was utilized as a surfactant. The third treatment was similar to the second treatment, in addition to leguminous compost that was added at $1.2 \mathrm{~kg} \mathrm{~m}^{-2}$. The fourth treatment was as the second treatment, in addition to leguminous compost $\left(\mathrm{C}_{\mathrm{L}} ; 1.2 \mathrm{~kg} \mathrm{~m}^{-2}\right)$ containing bacteria (Bacillus subtilis) as a biocompost fertilizer $\left(C_{L} B\right)$. The bacteria were prepared in a microbiological laboratory and added to the $\mathrm{C}_{\mathrm{L}}$ at approximately $0.6 \times 10^{9} \mathrm{CFU} \mathrm{g} \mathrm{g}^{-1}$ along with humic acid at $0.01 \%$ and amino acids at $0.01 \%$ to form a $C_{L} B$. The experimental treatments are summarized in Table 1.

Table 1. The description of the experimental fertilization treatments for soil and plant.

\begin{tabular}{ll}
\hline \multicolumn{1}{c}{ Treatment } & \multicolumn{1}{c}{ Description } \\
\hline $\begin{array}{l}\text { 1. } \mathrm{S}_{\mathrm{CF} 100} \\
\text { 2. } \mathrm{S}_{\mathrm{CF} 75}+\mathrm{F}_{\mathrm{CF} 25}\end{array}$ & $\begin{array}{l}\text { 100\% of the recommended NPK doses were applied to the soil as a control. } \\
75 \% \text { of the recommended NPK doses were applied to the soil, }+25 \% \text { of the recommended NPK doses } \\
\text { were applied as three foliar sprays (each spraying with the third of the } 25 \% \text { ). } \\
75 \% \text { of the recommended NPK doses were applied to the soil, }+25 \% \text { of the recommended NPK doses } \\
\text { were applied as three foliar sprays (each spraying with the third of the } 25 \% \text { ) }+1.2 \mathrm{~kg} \text { leguminous } \\
\text { compost } \mathrm{m}^{-2} \text {. }\end{array}$ \\
$\begin{array}{l}\text { 75\% of the recommended NPK doses were applied to the soil, }+25 \% \text { of the recommended NPK doses } \\
\text { were applied as three foliar sprays (each spraying with the third of the } 25 \%)+1.2 \mathrm{~kg} \text { biocompost } \\
\text { (leguminous compost containing bacteria; Bacillus subtilis) } \mathrm{m}^{-2} \text {. }\end{array}$ \\
\hline
\end{tabular}

All soil additions were spread manually on the soil surface after mixing with an appropriate amount of sand, then incorporated into the $20 \mathrm{~cm}$ upper layer. The treatments were secured for a randomized complete block design with ten unit $\left(10.5 \mathrm{~m}^{2}\right)$ replicates for each of the four treatments in both 2019 and 2020 seasons. All other practices recommended for standard cultivation for commercial Phaseolus vulgaris production were followed [32].

\subsection{Compost Preparation}

Twenty-five $\mathrm{kg}$ of green shoots of faba bean were incorporated into a similar weight containing different organic materials, including $0.5 \mathrm{~kg}$ and $0.5 \mathrm{~kg}$ of bulking agents and potassium humate $(\mathrm{K}-\mathrm{H})$, respectively, along with $12.0 \mathrm{~kg}$ and $12.0 \mathrm{~kg}$ of cattle manure and Egyptian clover plants, respectively, as $\mathrm{N}$ sources. For the compost mixture, green shoots of faba bean, bulking agents, potassium humate, cattle manure, and Egyptian clover plants contributed 50.0, 0.5, 0.5, 24.0, and 24.0\%, respectively. After mixing well, these mixtures were composted in a pilot plant utilizing the turning-pile system in trapezoidal piles (dimensions of the base were $5.0 \times 20.0 \times 7.5 \mathrm{~m}$ in height, length, and width, respectively). From August to February, during the bio-oxidative phase, overturning was performed every two weeks for the piles, keeping the level of moisture in the range of $40-60 \%$ while 
monitoring the temperature. The obtained compost was analyzed, and the resulting data were as follows: $\mathrm{pH}=7.5, \mathrm{EC}=2.1 \mathrm{dS} \mathrm{m}^{-1}$, content of organic matter $=19.6 \%$, $\mathrm{K}=152 \mathrm{~g} \mathrm{~kg}^{-1}, \mathrm{P}=33 \mathrm{~g} \mathrm{~kg}^{-1}$, and $\mathrm{N}=115 \mathrm{~g} \mathrm{~kg}^{-1}$.

\subsection{Soil Sampling}

Before sowing and after harvesting the Phaseolus vulgaris crop, three samples were randomly gathered from the 0-20 cm upper soil layer of three randomly selected experimental units and immediately transported to the laboratory and sieved with a $2 \mathrm{~mm}$ stainless-steel soil sampler.

\subsection{Assessments of Soil CEC, Organic Matter, and Nutrient Contents}

The procedures detailed in $[30,31]$ were applied to evaluate each of cation exchange capacity (CEC), organic matter (\%), $\mathrm{CaCO}_{3}(\%)$, and nutrient contents (e.g., N, P, K, Fe, Mn, and $\mathrm{Zn})$.

\subsection{Soil Organic Carbon and Soil Enzyme Activity Determinations}

Soil samples with a size of up to $2 \mathrm{~mm}$ were utilized to assess the organic $C$ content and enzyme activities in the tested soil. The samples were randomly gathered from all experimental units of all tested treatments, including the comparison units. The wet oxidation-redox titration method of Carter and Gregorich [33] was applied to determine organic C content. Cellulases were determined as the system of an enzyme, by which cellulose was degraded and reducing sugars were released as the end product. Cellulase activity was expressed as $\mathrm{mg}$ sugars released $\mathrm{g}^{-1}$ dry soil, $37^{\circ} \mathrm{C}, 24 \mathrm{~h}$. Then, the procedure (anthrone colorimetric analysis) of Hope and Burns [34] was applied to determine the reducing sugars. The procedures detailed in both Schinner and von Mersi [35] and Miller [36] were applied to assay soil invertase (EC 3.2.1.26) activity (mg sugars released $\mathrm{g}^{-1}$ dry soil, $37^{\circ} \mathrm{C}, 24 \mathrm{~h}$ ) and the content of reducing sugars. The buffered procedure of Kandeler and Gerber [37] was applied to assay soil urease (EC 3.5.1.5) activity ( $\mu g \mathrm{NH}_{3}-\mathrm{N}$ $\mathrm{g}^{-1}$ dry soil, $37^{\circ} \mathrm{C}, 24 \mathrm{~h}$ ). The back-titrating residual $\mathrm{H}_{2} \mathrm{O}_{2}$ with the $\mathrm{KMnO}_{4}$ procedure of Johnson and Temple [38] and Stepniewska et al. [39] had functioned to assay soil catalase (EC 1.11.1.6) activity ( $\mu \mathrm{mol} \mathrm{H}_{2} \mathrm{O}_{2} \mathrm{~g}^{-1}$ dry soil, $25^{\circ} \mathrm{C}, 24 \mathrm{~h}$ ).

Soil enzyme activities were evaluated using controls made by mixing the buffer solution with either the soil fractions or the substrate solution. The values were corrected by subtracting the combined absorbance values of the sample and substrate controls from those of the analytical samples.

\subsection{Assessment of Growth and Yield Components}

Fifty days after sowing, shoots of 10 plants were randomly gathered from each treatment (10 experimental units; one plant from each unit) to evaluate fresh weight (g), and after drying these shoots at $70{ }^{\circ} \mathrm{C}$, dry weight was recorded after two or more constant weights of each shoot.

Sixty-two to 70 days after sowing (green pod marketing stage), 10 plants from each treatment (10 experimental units; one plant from each unit) were randomly assigned to assess the average pod weight (g) and total plant green pods (g). Eighty days after sowing (dry seed marketing stage), the remaining plants were applied for dry seeds' weight (g per plant) after pods were picked to air dry for $3 \mathrm{~d}$.

\subsection{Leafy Nutrient Contents}

Uniform leafy samples were collected from 10 plants randomly gathered from each treatment (10 experimental units; one plant from each unit) to be dried (at $70{ }^{\circ} \mathrm{C}$ for $72 \mathrm{~h}$ ) and digested to assess leafy content of nutrients. The micro-Kjeldahl technique was applied to assess total $\mathrm{N}$ content ( $\left.\mathrm{mg} \mathrm{g}^{-1} \mathrm{DW}\right)$. The content $\left(\mathrm{mg} \mathrm{g}^{-1} \mathrm{DW}\right)$ of $\mathrm{P}$ was colorimetrically evaluated with the use of the reagent of stannous chloride-ammonium molybdate [40], following the extraction with $\mathrm{NaHCO}^{3}$ [41]. The flame photometer (ELE Flame Photometer, 
Leighton Buzzard, UK) technique was utilized to assess the content $\left(\mathrm{mg} \mathrm{g}^{-1} \mathrm{DW}\right)$ of $\mathrm{K}^{+}$. Besides this, the atomic absorption spectrophotometry apparatus was utilized to determine the contents ( $\mathrm{mg} \mathrm{g}^{-1} \mathrm{DW}$ ) of $\mathrm{Fe}^{2+}, \mathrm{Mn}^{2+}$, and $\mathrm{Zn}^{2+}$ nutrients spectrophotometry [42].

\subsection{Total Chlorophyll, Osmoprotectants, and Antioxidants Contents}

The total chlorophyll content was spectrophotometrically (UV-160A, Spectrometer, Shimadzu, Kyoto, Japan) evaluated applying the method of [43], utilizing leaf discs ( $0.5 \mathrm{~g})$. The leafy samples were homogenized with acetone solution $(80 \%, v / v)$ and then the centrifugation process was practiced $(3000 \times g, 20 \mathrm{~min})$ for obtaining the supernatant to measure the absorbances on 470, 645, and $663 \mathrm{~nm}$.

At the field level, standardized, uniform leaves were assigned to measure chlorophyll fluorescence at $360 \mu \mathrm{mol} \mathrm{mol}{ }^{-1} \mathrm{CO}_{2}, 21^{\circ} \mathrm{C}$, and $600 \mu \mathrm{mol} \mathrm{m}^{-2} \mathrm{~s}^{-1}$ PPFD utilizing a potable pulse-modulated fluorometer (FMS-2, Hansatech, Norfolk, UK) according to the procedure of Li et al. [44]. The maximum quantum yield of photosystem II (PSII) (Fv/Fm) was computed using the Maxwell and Johnson [45] formulae. According to the equal absorption, photosynthesis $\mathrm{PI}_{\mathrm{ABS}}$ was computed according to the formula of Clark et al. [46].

Phaseolus vulgaris leaf RWC was evaluated applying the procedure of Osman and Rady [47] with the use of leafy discs $(n=20)$ after exclusion of the midrib. The fresh, turgid, and dry mass were recorded for applying in the following formula:

$$
\text { RWC }(\%)=[(\text { fresh mass }- \text { dry mass }) /(\text { turgid mass }- \text { dry mass })] \times 100
$$

The Irigoyen et al. [48] method was used (using ethyl alcohol $96 \%, v / v$ ) to extract and determine soluble sugar content $\left(\mathrm{mg} \mathrm{g}^{-1} \mathrm{DW}\right)$ in the standardized, uniform leafy samples. The same leafy tissues were utilized to determine the content of free proline ( $\mu \mathrm{g} \mathrm{g}^{-1} \mathrm{DW}$ ) following the Bates et al. [49] method. Besides this, the full procedures of Griffith [50] and Mukherjee and Choudhari [51] were applied for determining glutathione (nmol glutathione $\mathrm{g}^{-1} \mathrm{FW}$ ) and ascorbate $\left(\mu \mathrm{mol} \mathrm{g}^{-1} \mathrm{FW}\right.$ ) contents, respectively.

\subsection{Assaying the Activities of Antioxidative Enzymes}

Leafy samples (from all treatments, $0.5 \mathrm{~g}$ ) were extracted applying the full Mukherjee and Choudhari [51] procedure. After centrifuging the homogenates $(15,000 \times g, 10 \mathrm{~min})$ the obtained supernatants were utilized to assay enzyme activities (guaiacol peroxidase (GPD), catalase (CAT), and superoxide dismutase (SOD)). The full procedures of Giannopolitis and Ries [52], Aebi [53], and Putter [54] were applied for assaying SOD (EC1.15.1.1), CAT (EC1.11.1.6), and GPD activities, respectively. For enzyme, nitro blue tetrazolium (NBT), $\mathrm{H}_{2} \mathrm{O}_{2}$, and guaiacol with $\mathrm{H}_{2} \mathrm{O}_{2}$ solutions were the substrates utilized, respectively. Besides this, the full method of [55] was applied for assessing the protein content.

\subsection{Data Analysis}

Analysis of data was performed with IBM ${ }^{\circledR}$ SPSS $^{\circledR}$ (SPSS Inc., IBM Corporation, New York, NY, USA) Statistics Version 25 (2017) for Windows. To verify the normal distribution of data the Shapiro-Wilk test was used [56,57] for main effects and interactions. Due to the data having normal distribution $(p \leq 0.05)$, parametric statistical tests were applied. The two-way ANOVA was performed to highlight the effect of four fertilization treatments, two growing seasons, and the interaction between fertilization treatments and growing seasons. Two-way ANOVA, followed by a post hoc LSD test was used for multiple comparisons among means of fertilization treatments, growing seasons, and the interaction between fertilization treatments and growing seasons. All statistical tests were two-tailed, and a $p$-value less than or equal to 0.05 was considered statistically significant. A confidence interval was estimated at $95 \%$. 


\section{Results}

3.1. Soil Physical and Chemical Properties Response to $S_{C F 75}+F_{C F 25}+C_{L} B$ Supplementation for Two Seasons (2019 and 2020)

Data in Table 2 show the desired changes in soil characteristics during the two seasons (2019 and 2020) resulting from soil supplementation with $\mathrm{S}_{\mathrm{CF} 75}+\mathrm{F}_{\mathrm{CF} 25}+\mathrm{C}_{\mathrm{L}} \mathrm{B}$ (chemical fertilizers applied to the soil at $75 \%$ of recommended NPK doses + chemical fertilizers applied as foliar sprays at $25 \%$ of recommended NPK doses + biocompost; leguminous compost $\left(1.2 \mathrm{~kg} \mathrm{~m}^{-2}\right)$ containing bacteria) as the best treatment. The soil composition of clay, silt, and sand was not changed in either of the 2019 and 2020 growing seasons with $\mathrm{S}_{\mathrm{CF} 75}+\mathrm{F}_{\mathrm{CF} 25}+\mathrm{C}_{\mathrm{L}} \mathrm{B}$ application. Other characteristics (e.g., $\mathrm{pH}, \mathrm{EC}$, and $\mathrm{CaCO}_{3}$ content) were slightly affected.

Table 2. The physicochemical properties of the tested soil before sowing and following and after harvest.

\begin{tabular}{|c|c|c|c|c|}
\hline \multirow{3}{*}{ Parameters } & \multicolumn{4}{|c|}{ Properties } \\
\hline & \multicolumn{2}{|c|}{2019} & \multicolumn{2}{|c|}{2020} \\
\hline & Before Sowing & After Harvest & Before Sowing & After Harvest \\
\hline Clay & $8.2 \pm 0.6^{*}$ & $8.2 \pm 0.5$ & $8.2 \pm 0.7$ & $8.3 \pm 0.8$ \\
\hline Silt & $10.7 \pm 0.8$ & $10.8 \pm 0.8$ & $10.8 \pm 0.7$ & $10.9 \pm 1.0$ \\
\hline Sand & $81.1 \pm 7.2$ & $81.0 \pm 6.9$ & $81.0 \pm 6.7$ & $80.8 \pm 6.2$ \\
\hline Soil texture & Loamy Sand & Loamy Sand & Loamy Sand & Loamy Sand \\
\hline $\mathrm{pH}$ & $8.35 \pm 0.51$ & $8.12 \pm 0.48$ & $8.02 \pm 0.42$ & $7.82 \pm 0.38$ \\
\hline $\mathrm{EC}\left(\mathrm{dS} \mathrm{m}^{-1}\right)$ & $3.20 \pm 0.24$ & $3.24 \pm 0.24$ & $3.31 \pm 0.26$ & $3.44 \pm 0.25$ \\
\hline $\mathrm{CaCO}_{3}(\%)$ & $8.62 \pm 0.54$ & $8.52 \pm 0.55$ & $8.44 \pm 0.50$ & $8.10 \pm 0.48$ \\
\hline \multicolumn{5}{|c|}{ Available nutrients ( $\mathrm{mg} \mathrm{kg}^{-1}$ soil) before sowing } \\
\hline $\mathrm{N}$ & \multicolumn{2}{|c|}{$12.7 \pm 0.2$} & \multicolumn{2}{|c|}{$27.8 \pm 0.5$} \\
\hline $\mathrm{P}$ & \multicolumn{2}{|c|}{$7.6 \pm 0.1$} & \multicolumn{2}{|c|}{$15.3 \pm 0.3$} \\
\hline K & \multicolumn{2}{|c|}{$16.4 \pm 0.3$} & \multicolumn{2}{|c|}{$40.1 \pm 0.8$} \\
\hline
\end{tabular}

*Values $\pm \mathrm{SE}, \mathrm{dS} \mathrm{m}^{-1}=$ deciSiemens per meter, and $\mathrm{S}_{\mathrm{CF} 75}+\mathrm{F}_{\mathrm{CF} 25}+\mathrm{C}_{\mathrm{L}} \mathrm{B}=$ chemical fertilizers applied to the soil at $75 \%$ of recommended NPK doses + chemical fertilizers applied as foliar sprays at $25 \%$ of recommended NPK doses + biocompost; leguminous compost $\left(1.2 \mathrm{~kg} \mathrm{~m}^{-2}\right)$ containing bacteria.

\subsection{Response of Soil Chemical Properties and Plant Performances to the Growing Season}

The growing season as the main factor in this study affected the chemical properties of the tested soil, soil and plant contents of essential nutrients, activities of various enzymes, plant growth and productivity, and plant physiobiochemistry. The 2020 growing season significantly outperformed the 2019 growing season in terms of all the examined parameters of the soil and Phaseolus vulgaris plant, which are presented in Tables 3-6 and Figures 1-12.

Table 3. Response of soil nutrient contents to growing season (2019 and 2020) and different fertilization strategies applied to virgin sandy soil.

\begin{tabular}{|c|c|c|c|}
\hline \multirow{2}{*}{ Source of Variation } & \multicolumn{3}{|c|}{ Available Macronutrient Contents (mg kg-1 Soil) } \\
\hline & Available N & Available P & Available K \\
\hline Season $(S)$ & * & ns & * \\
\hline 2019 & $30.3 \pm 0.2^{b}$ & $17.5 \pm 0.2^{\mathrm{a}}$ & $37.5 \pm 0.3^{b}$ \\
\hline 2020 & $35.9 \pm 0.3^{a}$ & $17.5 \pm 0.2^{\mathrm{a}}$ & $45.5 \pm 0.4^{\mathrm{a}}$ \\
\hline Fertilization $(\mathrm{F})$ & * & $* *$ & $* *$ \\
\hline $\mathrm{S}_{\mathrm{CF} 100}$ & $27.5 \pm 0.3^{c}$ & $11.8 \pm 0.1^{\mathrm{c}}$ & $34.3 \pm 0.4^{\mathrm{c}}$ \\
\hline $\mathrm{S}_{\mathrm{CF} 75}+\mathrm{F}_{\mathrm{CF} 25}$ & $27.1 \pm 0.2^{c}$ & $11.5 \pm 0.1^{\mathrm{c}}$ & $33.4 \pm 0.3^{c}$ \\
\hline $\mathrm{S}_{\mathrm{CF} 75}+\mathrm{F}_{\mathrm{CF} 25}+\mathrm{C}_{\mathrm{L}}$ & $34.9 \pm 0.3^{b}$ & $17.5 \pm 0.2^{b}$ & $43.9 \pm 0.4^{b}$ \\
\hline $\mathrm{S}_{\mathrm{CF} 75}+\mathrm{F}_{\mathrm{CF} 25}+\mathrm{C}_{\mathrm{L}} \mathrm{B}$ & $43.1 \pm 0.4^{\mathrm{a}}$ & $21.8 \pm 0.2^{\mathrm{a}}$ & $54.5 \pm 0.4^{\mathrm{a}}$ \\
\hline
\end{tabular}


Table 3. Cont.

\begin{tabular}{|c|c|c|c|}
\hline \multirow{2}{*}{ Source of Variation } & \multicolumn{3}{|c|}{ Available Macronutrient Contents (mg kg ${ }^{-1}$ Soil) } \\
\hline & Available N & Available P & Available K \\
\hline $\mathrm{S} \times \mathrm{F}$ & * & ** & ** \\
\hline $2019 \times S_{C F 100}$ & $25.6 \pm 0.2^{d}$ & $10.4 \pm 0.1^{\mathrm{e}}$ & $31.2 \pm 0.3^{\mathrm{d}}$ \\
\hline $2019 \times \mathrm{S}_{\mathrm{CF} 75}+\mathrm{F}_{\mathrm{CF} 25}$ & $24.8 \pm 0.2^{\mathrm{d}}$ & $10.0 \pm 0.1^{\mathrm{e}}$ & $29.9 \pm 0.3^{d}$ \\
\hline $2019 \times \mathrm{S}_{\mathrm{CF} 75}+\mathrm{F}_{\mathrm{CF} 25}+\mathrm{C}_{\mathrm{L}}$ & $31.4 \pm 0.2^{\mathrm{c}}$ & $15.3 \pm 0.1^{\mathrm{c}}$ & $39.4 \pm 0.3^{c}$ \\
\hline $2019 \times \mathrm{S}_{\mathrm{CF} 75}+\mathrm{F}_{\mathrm{CF} 25}+\mathrm{C}_{\mathrm{L}} \mathrm{B}$ & $39.5 \pm 0.3^{b}$ & $19.4 \pm 0.2^{b}$ & $49.3 \pm 0.4^{b}$ \\
\hline $2020 \times S_{C F 100}$ & $29.4 \pm 0.3^{c}$ & $13.2 \pm 0.1^{\mathrm{d}}$ & $37.4 \pm 0.4^{\mathrm{c}}$ \\
\hline $2020 \times \mathrm{S}_{\mathrm{CF} 75}+\mathrm{F}_{\mathrm{CF} 25}$ & $29.3 \pm 0.2^{c}$ & $12.9 \pm 0.1^{\mathrm{d}}$ & $36.8 \pm 0.3^{\mathrm{c}}$ \\
\hline $2020 \times \mathrm{S}_{\mathrm{CF} 75}+\mathrm{F}_{\mathrm{CF} 25}+\mathrm{C}_{\mathrm{L}}$ & $38.4 \pm 0.3^{b}$ & $19.6 \pm 0.2^{b}$ & $48.3 \pm 0.4^{\mathrm{b}}$ \\
\hline $2020 \times \mathrm{S}_{\mathrm{CF} 75}+\mathrm{F}_{\mathrm{CF} 25}+\mathrm{C}_{\mathrm{L}} \mathrm{B}$ & $46.6 \pm 0.4^{\mathrm{a}}$ & $24.1 \pm 0.2^{\mathrm{a}}$ & $59.6 \pm 0.4^{\mathrm{a}}$ \\
\hline \multirow{2}{*}{ Source of Variation } & \multicolumn{3}{|c|}{ Available Micronutrient Contents (mg kg ${ }^{-1}$ Soil) } \\
\hline & Available Fe & Available Mn & Available Zn \\
\hline Season (S) & * & * & * \\
\hline 2019 & $4.61 \pm 0.26^{b}$ & $3.13 \pm 0.16^{b}$ & $2.22 \pm 0.14^{b}$ \\
\hline 2020 & $6.08 \pm 0.37^{\mathrm{a}}$ & $4.56 \pm 0.24^{\mathrm{a}}$ & $3.16 \pm 0.16^{\mathrm{a}}$ \\
\hline Fertilization $(\mathrm{F})$ & $* *$ & * & * \\
\hline $\mathrm{S}_{\mathrm{CF} 100}$ & $4.09 \pm 0.25^{c}$ & $3.15 \pm 0.16^{\mathrm{c}}$ & $2.28 \pm 0.13^{c}$ \\
\hline $\mathrm{S}_{\mathrm{CF} 75}+\mathrm{F}_{\mathrm{CF} 25}$ & $4.11 \pm 0.27^{\mathrm{c}}$ & $3.10 \pm 0.16^{\mathrm{c}}$ & $2.24 \pm 0.11^{\mathrm{c}}$ \\
\hline $\mathrm{S}_{\mathrm{CF} 75}+\mathrm{F}_{\mathrm{CF} 25}+\mathrm{C}_{\mathrm{L}}$ & $5.70 \pm 0.33^{b}$ & $4.06 \pm 0.21^{\mathrm{b}}$ & $2.80 \pm 0.17^{\mathrm{b}}$ \\
\hline $\begin{array}{c}\mathrm{S}_{\mathrm{CF} 75}+\mathrm{F}_{\mathrm{CF} 25}+\mathrm{C}_{\mathrm{L}} \mathrm{B} \\
\mathrm{S} \times \mathrm{F}\end{array}$ & $\underset{* *}{7.47 \pm} 0.41^{\mathrm{a}}$ & $\begin{array}{c}5.08 \pm 0.26^{a} \\
*\end{array}$ & $\begin{array}{c}3.44 \pm 0.18^{\mathrm{a}} \\
*\end{array}$ \\
\hline $2019 \times \mathrm{S}_{\mathrm{CF} 100}$ & $3.52 \pm 0.22 \mathrm{~d}$ & $2.28 \pm 0.14^{\mathrm{d}}$ & $1.74 \pm 0.10^{\mathrm{d}}$ \\
\hline $2019 \times \mathrm{S}_{\mathrm{CF} 75}+\mathrm{F}_{\mathrm{CF} 25}$ & $3.64 \pm 0.23^{d}$ & $2.22 \pm 0.12^{\mathrm{d}}$ & $1.70 \pm 0.08^{\mathrm{d}}$ \\
\hline $2019 \times \mathrm{S}_{\mathrm{CF} 75}+\mathrm{F}_{\mathrm{CF} 25}+\mathrm{C}_{\mathrm{L}}$ & $4.48 \pm 0.25^{\mathrm{c}}$ & $3.55 \pm 0.16^{c}$ & $2.38 \pm 0.18^{c}$ \\
\hline $2019 \times \mathrm{S}_{\mathrm{CF} 75}+\mathrm{F}_{\mathrm{CF} 25}+\mathrm{C}_{\mathrm{L}} \mathrm{B}$ & $6.79 \pm 0.34^{b}$ & $4.48 \pm 0.22^{b}$ & $3.04 \pm 0.18^{b}$ \\
\hline $2020 \times S_{\text {CF100 }}$ & $4.66 \pm 0.27^{\mathrm{c}}$ & $4.02 \pm 0.18^{b c}$ & $2.82 \pm 0.16^{\mathrm{c}}$ \\
\hline $2020 \times \mathrm{S}_{\mathrm{CF} 75}+\mathrm{F}_{\mathrm{CF} 25}$ & $4.58 \pm 0.31^{\mathrm{c}}$ & $3.98 \pm 0.20 b c$ & $2.78 \pm 0.14^{\mathrm{c}}$ \\
\hline $2020 \times \mathrm{S}_{\mathrm{CF} 75}+\mathrm{F}_{\mathrm{CF} 25}+\mathrm{C}_{\mathrm{L}}$ & $6.92 \pm 0.40^{b}$ & $4.57 \pm 0.26^{\mathrm{b}}$ & $3.21 \pm 0.16^{b}$ \\
\hline $2020 \times \mathrm{S}_{\mathrm{CF} 75}+\mathrm{F}_{\mathrm{CF} 25}+\mathrm{C}_{\mathrm{L}} \mathrm{B}$ & $8.15 \pm 0.48^{\mathrm{a}}$ & $5.68 \pm 0.30^{\mathrm{a}}$ & $3.84 \pm 0.18^{a}$ \\
\hline
\end{tabular}

** and * indicate, respectively, differences at $p \leq 0.05$ and $p \leq 0.01$ probability level, respectively, and ns indicates not significant difference. Mean values with different letters in each column are significant $(p \leq 0.05)$. $\mathrm{S}_{\mathrm{CF} 100}=$ chemical fertilizers applied to the soil at full recommended NPK doses, $\mathrm{S}_{\mathrm{CF} 75}=$ chemical fertilizers applied to the soil at $75 \%$ of recommended NPK doses, $\mathrm{F}_{\mathrm{CF} 25}=$ chemical fertilizers applied as foliar sprays at $25 \%$ of recommended NPK doses, $C_{L}=1.2 \mathrm{~kg}$ leguminous compost $\mathrm{m}^{-2}$, and $\mathrm{C}_{\mathrm{L}} \mathrm{B}=$ biocompost; leguminous compost $\left(1.2 \mathrm{~kg}\right.$ per $\left.^{-2}\right)$ containing bacteria.

Table 4. Response of growth and productivity of Phaseolus vulgaris plants to growing season (2019 and 2020) and different fertilization strategies applied to virgin sandy soil.

\begin{tabular}{|c|c|c|c|c|}
\hline \multirow[b]{2}{*}{ Source of Variation } & \multicolumn{2}{|c|}{ Growth Parameters } & \multicolumn{2}{|c|}{ Yield Parameters } \\
\hline & $\begin{array}{c}\text { Shoot FW } \\
\left(\text { g Plant }^{-1}\right)\end{array}$ & $\begin{array}{l}\text { Shoot DW } \\
\left.\text { (g Plant }^{-1}\right)\end{array}$ & $\begin{array}{l}\text { Green Pod Weight } \\
\left(\text { ton ha } \mathrm{h}^{-1}\right)\end{array}$ & $\begin{array}{l}\text { Dry Seed Weight } \\
\quad\left(\text { ton } \mathrm{ha}^{-1}\right)\end{array}$ \\
\hline Season (S) & * & * & * & * \\
\hline 2019 & $18.3 \pm 1.7^{b}$ & $2.25 \pm 2.1^{b}$ & $2.62 \pm 0.28^{b}$ & $0.67 \pm 0.07^{b}$ \\
\hline 2020 & $22.1 \pm 2.2^{\mathrm{a}}$ & $2.60 \pm 2.6^{a}$ & $3.00 \pm 0.30^{\mathrm{a}}$ & $0.86 \pm 0.09^{\mathrm{a}}$ \\
\hline Fertilization $(\mathrm{F})$ & $* *$ & * & $*$ & $* *$ \\
\hline $\mathrm{S}_{\mathrm{CF} 100}$ & $15.3 \pm 1.4^{\mathrm{d}}$ & $1.95 \pm 2.1^{\mathrm{d}}$ & $2.16 \pm 0.23^{d}$ & $0.52 \pm 0.06^{\mathrm{d}}$ \\
\hline $\mathrm{S}_{\mathrm{CF} 75}+\mathrm{F}_{\mathrm{CF} 25}$ & $17.9 \pm 1.9^{c}$ & $2.22 \pm 2.1^{\mathrm{c}}$ & $2.62 \pm 0.26^{\mathrm{c}}$ & $0.63 \pm 0.07^{c}$ \\
\hline $\mathrm{S}_{\mathrm{CF} 75}+\mathrm{F}_{\mathrm{CF} 25}+\mathrm{C}_{\mathrm{L}}$ & $21.6 \pm 2.1^{b}$ & $2.59 \pm 2.6^{b}$ & $3.07 \pm 0.32^{b}$ & $0.83 \pm 0.08^{b}$ \\
\hline $\begin{array}{c}\mathrm{S}_{\mathrm{CF} 75}+\mathrm{F}_{\mathrm{CF} 25}+\mathrm{C}_{\mathrm{L}} \mathrm{B} \\
\mathrm{S} \times \mathrm{F}\end{array}$ & $26.1 \pm \underset{* *}{ } 2.5^{\mathrm{a}}$ & $2.95 \pm 2.8^{a}$ & $3.39 \pm 0.34^{\mathrm{a}}$ & $1.09 \pm \underset{* *}{0.11^{\mathrm{a}}}$ \\
\hline $2019 \times S_{\mathrm{CF} 100}$ & $14.2 \pm 1.3^{\mathrm{e}}$ & $1.79 \pm 1.8^{\mathrm{e}}$ & $1.96 \pm 0.21^{\mathrm{e}}$ & $0.46 \pm 0.05^{\mathrm{e}}$ \\
\hline $2019 \times \mathrm{S}_{\mathrm{CF} 75}+\mathrm{F}_{\mathrm{CF} 25}$ & $16.6 \pm 1.7^{\mathrm{d}}$ & $2.09 \pm 1.9^{\mathrm{d}}$ & $2.41 \pm 0.25^{d}$ & $0.55 \pm 0.05^{\mathrm{d}}$ \\
\hline $2019 \times \mathrm{S}_{\mathrm{CF} 75}+\mathrm{F}_{\mathrm{CF} 25}+\mathrm{C}_{\mathrm{L}}$ & $19.0 \pm 1.8^{\mathrm{c}}$ & $2.36 \pm 2.2^{c}$ & $2.90 \pm 0.31^{c}$ & $0.72 \pm 0.07^{c}$ \\
\hline $2019 \times \mathrm{S}_{\mathrm{CF} 75}+\mathrm{F}_{\mathrm{CF} 25}+\mathrm{C}_{\mathrm{L}} \mathrm{B}$ & $23.4 \pm 2.1^{b}$ & $2.75 \pm 2.5^{b}$ & $3.19 \pm 0.34^{b}$ & $0.96 \pm 0.10^{b}$ \\
\hline
\end{tabular}


Table 4. Cont.

\begin{tabular}{|c|c|c|c|c|}
\hline \multirow[b]{2}{*}{ Source of Variation } & \multicolumn{2}{|c|}{ Growth Parameters } & \multicolumn{2}{|c|}{ Yield Parameters } \\
\hline & $\begin{array}{l}\text { Shoot FW } \\
\left(\text { g Plant }^{-1}\right)\end{array}$ & $\begin{array}{l}\text { Shoot DW } \\
\left(\text { g Plant }^{-1}\right)\end{array}$ & $\begin{array}{c}\text { Green Pod Weight } \\
\left(\text { ton } \mathrm{ha}^{-1}\right)\end{array}$ & $\begin{array}{l}\text { Dry Seed Weight } \\
\left(\text { ton } \mathrm{ha}^{-1}\right)\end{array}$ \\
\hline $2020 \times S_{C F 100}$ & $16.4 \pm 1.5^{\mathrm{d}}$ & $2.11 \pm 2.3^{\mathrm{d}}$ & $2.36 \pm 0.25^{d}$ & $0.57 \pm 0.07^{\mathrm{d}}$ \\
\hline $2020 \times \mathrm{S}_{\mathrm{CF} 75}+\mathrm{F}_{\mathrm{CF} 25}$ & $19.1 \pm 2.0^{c}$ & $2.34 \pm 2.3^{c}$ & $2.83 \pm 0.27^{c}$ & $0.69 \pm 0.08^{c}$ \\
\hline $2020 \times \mathrm{S}_{\mathrm{CF} 75}+\mathrm{F}_{\mathrm{CF} 25}+\mathrm{C}_{\mathrm{L}}$ & $24.2 \pm 2.3^{b}$ & $2.81 \pm 2.9^{b}$ & $3.24 \pm 0.32^{b}$ & $0.94 \pm 0.08^{b}$ \\
\hline $2020 \times \mathrm{S}_{\mathrm{CF} 75}+\mathrm{F}_{\mathrm{CF} 25}+\mathrm{C}_{\mathrm{L}} \mathrm{B}$ & $28.7 \pm 2.8^{a}$ & $3.14 \pm 3.0^{\mathrm{a}}$ & $3.58 \pm 0.34^{\mathrm{a}}$ & $1.22 \pm 0.11^{\mathrm{a}}$ \\
\hline
\end{tabular}

** and * indicate, respectively, differences at $p \leq 0.05$ and $p \leq 0.01$ probability level, respectively. Mean values with different letters in each column are significant $(p \leq 0.05) . \mathrm{S}_{\mathrm{CF} 100}=$ chemical fertilizers applied to the soil at full recommended NPK doses, $\mathrm{S}_{\mathrm{CF} 75}=$ chemical fertilizers applied to the soil at $75 \%$ of recommended NPK doses, $\mathrm{F}_{\text {CF25 }}=$ chemical fertilizers applied as foliar sprays at $25 \%$ of recommended NPK doses, $C_{\mathbf{L}}=1.2 \mathrm{~kg}$ leguminous compost $\mathrm{m}^{-2}$, and $\mathrm{C}_{\mathbf{L}} \mathrm{B}=$ biocompost; leguminous compost $\left(1.2 \mathrm{~kg} \mathrm{~m}^{-2}\right)$ containing bacteria.

Table 5. Response of nutritional contents of Phaseolus vulgaris plants to growing season (2019 and 2020) and different fertilization strategies applied to virgin sandy soil.

\begin{tabular}{|c|c|c|c|c|c|c|}
\hline \multirow{2}{*}{ Source of Variation } & \multicolumn{3}{|c|}{ Macronutrient Contents (mg g $\left.{ }^{-1} \mathrm{DW}\right)$} & \multicolumn{3}{|c|}{ Micronutrient Contents (mg kg $\left.{ }^{-1} \mathrm{DW}\right)$} \\
\hline & $\mathbf{N}$ & $\mathbf{P}$ & K & $\mathrm{Fe}$ & Mn & $\mathrm{Zn}$ \\
\hline Season (S) & * & * & * & * & * & * \\
\hline 2019 & $21.1 \pm 0.5^{b}$ & $1.93 \pm 0.05^{b}$ & $24.0 \pm 0.6^{b}$ & $274 \pm 14^{\mathrm{b}}$ & $180 \pm 7^{b}$ & $126 \pm 4^{b}$ \\
\hline 2020 & $25.3 \pm 0.8^{a}$ & $2.42 \pm 0.07^{\mathrm{a}}$ & $27.8 \pm 0.8^{a}$ & $322 \pm 17^{\mathrm{a}}$ & $223 \pm 9^{a}$ & $151 \pm 5^{a}$ \\
\hline Fertilization (F) & $*$ & $* *$ & $*$ & * & $* *$ & $* *$ \\
\hline $\mathrm{S}_{\mathrm{CF} 100}$ & $16.9 \pm 0.5^{\mathrm{d}}$ & $1.47 \pm 0.04^{\mathrm{d}}$ & $19.4 \pm 0.5^{\mathrm{d}}$ & $221 \pm 12^{d}$ & $144 \pm 6^{\mathrm{d}}$ & $100 \pm 4^{\mathrm{d}}$ \\
\hline $\mathrm{S}_{\mathrm{CF} 75}+\mathrm{F}_{\mathrm{CF} 25}$ & $20.9 \pm 0.6^{c}$ & $1.89 \pm 0.05^{c}$ & $23.6 \pm 0.6^{c}$ & $265 \pm 14^{c}$ & $176 \pm 7^{c}$ & $127 \pm 5^{c}$ \\
\hline $\mathrm{S}_{\mathrm{CF} 75}+\mathrm{F}_{\mathrm{CF} 25}+\mathrm{C}_{\mathrm{L}}$ & $25.6 \pm 0.7^{b}$ & $2.43 \pm 0.07^{b}$ & $28.1 \pm 0.7^{b}$ & $327 \pm 17^{b}$ & $222 \pm 9 b$ & $152 \pm 5^{b}$ \\
\hline $\begin{array}{c}\mathrm{S}_{\mathrm{CF} 75}+\mathrm{F}_{\mathrm{CF} 25}+\mathrm{C}_{\mathrm{L}} \mathrm{B} \\
\mathrm{S} \times \mathrm{F}\end{array}$ & $29.3 \pm 0.8^{a}$ & $2.92 \pm \underset{* *}{0.09^{a}}$ & $32.6 \pm 0.9^{a}$ & $\begin{array}{c}378 \pm 21^{a} \\
*\end{array}$ & $264 \underset{* *}{ \pm} 10^{a}$ & $176 \pm 6^{a}$ \\
\hline $2019 \times S_{C F 100}$ & $15.2 \pm 0.4^{\mathrm{e}}$ & $1.22 \pm 0.03^{\mathrm{e}}$ & $17.8 \pm 0.3^{\mathrm{e}}$ & $198 \pm 10^{\mathrm{e}}$ & $128 \pm 5^{\mathrm{e}}$ & $89 \pm 3^{e}$ \\
\hline $2019 \times \mathrm{S}_{\mathrm{CF} 75}+\mathrm{F}_{\mathrm{CF} 25}$ & $18.4 \pm 0.5^{\mathrm{d}}$ & $1.68 \pm 0.04^{\mathrm{d}}$ & $21.1 \pm 0.5^{\mathrm{d}}$ & $239 \pm 12^{d}$ & $156 \pm 6^{d}$ & $112 \pm 4^{\mathrm{d}}$ \\
\hline $2019 \times \mathrm{S}_{\mathrm{CF} 75}+\mathrm{F}_{\mathrm{CF} 25}+\mathrm{C}_{\mathrm{L}}$ & $23.1 \pm 0.5^{\mathrm{c}}$ & $2.14 \pm 0.06^{c}$ & $26.4 \pm 0.6^{\mathrm{c}}$ & $298 \pm 15^{c}$ & $192 \pm 8^{c}$ & $136 \pm 4^{c}$ \\
\hline $2019 \times \mathrm{S}_{\mathrm{CF} 75}+\mathrm{F}_{\mathrm{CF} 25}+\mathrm{C}_{\mathrm{L}} \mathrm{B}$ & $27.6 \pm 0.6^{b}$ & $2.67 \pm 0.08^{b}$ & $30.6 \pm 0.8^{b}$ & $360 \pm 20^{b}$ & $244 \pm 9^{b}$ & $165 \pm 5^{b}$ \\
\hline $2020 \times S_{C F 100}$ & $18.6 \pm 0.6^{\mathrm{d}}$ & $1.72 \pm 0.05^{\mathrm{d}}$ & $20.9 \pm 0.6^{d}$ & $244 \pm 14^{\mathrm{d}}$ & $160 \pm 7^{d}$ & $110 \pm 4^{\mathrm{d}}$ \\
\hline $2020 \times \mathrm{S}_{\mathrm{CF} 75}+\mathrm{F}_{\mathrm{CF} 25}$ & $23.4 \pm 0.7^{\mathrm{c}}$ & $2.10 \pm 0.05^{\mathrm{c}}$ & $26.0 \pm 0.6^{\mathrm{c}}$ & $290 \pm 16^{c}$ & $196 \pm 7^{c}$ & $141 \pm 5^{c}$ \\
\hline $2020 \times \mathrm{S}_{\mathrm{CF} 75}+\mathrm{F}_{\mathrm{CF} 25}+\mathrm{C}_{\mathrm{L}}$ & $28.0 \pm 0.9^{b}$ & $2.71 \pm 0.07^{b}$ & $29.8 \pm 0.8^{b}$ & $356 \pm 18^{b}$ & $251 \pm 9^{b}$ & $168 \pm 5^{b}$ \\
\hline $2020 \times \mathrm{S}_{\mathrm{CF} 75}+\mathrm{F}_{\mathrm{CF} 25}+\mathrm{C}_{\mathrm{L}} \mathrm{B}$ & $31.0 \pm 0.9^{\mathrm{a}}$ & $3.16 \pm 0.09^{\mathrm{a}}$ & $34.6 \pm 1.0^{\mathrm{a}}$ & $396 \pm 20^{a}$ & $284 \pm 11^{\mathrm{a}}$ & $186 \pm 7^{a}$ \\
\hline
\end{tabular}

** and * indicate, respectively, differences at $p \leq 0.05$ and $p \leq 0.01$ probability level, respectively. Mean values with different letters in each column are significant $(p \leq 0.05)$. $\mathrm{S}_{\mathrm{CF} 100}=$ chemical fertilizers applied to the soil at full recommended NPK doses, $\mathrm{S}_{\mathrm{CF} 75}=$ chemical fertilizers applied to the soil at $75 \%$ of recommended NPK doses, $\mathrm{F}_{\text {CF25 }}=$ chemical fertilizers applied as foliar sprays at $25 \%$ of recommended NPK doses, $C_{L}=1.2 \mathrm{~kg}$ leguminous compost $\mathrm{m}^{-2}$, and $\mathrm{C}_{\mathrm{L}} \mathrm{B}=$ biocompost; leguminous compost $\left(1.2 \mathrm{~kg} \mathrm{~m}^{-2}\right)$ containing bacteria.

Table 6. Response of photosynthetic parameters, relative water content, and osmoprotectant contents of Phaseolus vulgaris plants to growing season (2019 and 2020) and different fertilization strategies applied to virgin sandy soil.

\begin{tabular}{|c|c|c|c|c|c|c|}
\hline \multirow{2}{*}{ Source of Variation } & \multicolumn{3}{|c|}{ Photosynthetic Parameters } & \multicolumn{3}{|c|}{$\begin{array}{c}\text { Leaf Relative Water Content (RWC) and } \\
\text { Osmoprotectant Contents }\end{array}$} \\
\hline & $\begin{array}{l}\text { TChl Content } \\
\left(\mathrm{g} \mathrm{kg}^{-1} \text { FW) }\right.\end{array}$ & Fv/Fm & PI (\%) & RWC (\%) & $\begin{array}{c}\text { Proline } \\
\left(\mu g^{-1} \mathrm{DW}\right)\end{array}$ & $\begin{array}{c}\text { TS Sugars } \\
\left(\mathrm{mg} \mathrm{g}^{-1} \mathrm{DW}\right)\end{array}$ \\
\hline Season $(S)$ & * & * & * & * & * & * \\
\hline 2019 & $1.76 \pm 0.03^{b}$ & $0.77 \pm 0.02^{b}$ & $13.7 \pm 0.3^{b}$ & $65.0 \pm 2.3^{b}$ & $160 \pm 5^{b}$ & $18.0 \pm 0.4^{b}$ \\
\hline 2020 & $2.14 \pm 0.04^{\mathrm{a}}$ & $0.83 \pm 0.03^{a}$ & $15.9 \pm 0.4^{\mathrm{a}}$ & $73.8 \pm 2.5^{\mathrm{a}}$ & $185 \pm 6^{\mathrm{a}}$ & $22.4 \pm 0.5^{\mathrm{a}}$ \\
\hline Fertilization (F) & $* *$ & * & * & $*$ & $* *$ & $* *$ \\
\hline $\mathrm{S}_{\mathrm{CF} 100}$ & $1.30 \pm 0.03^{\mathrm{d}}$ & $0.71 \pm 0.02^{\mathrm{d}}$ & $11.5 \pm 0.3^{\mathrm{d}}$ & $56.7 \pm 2.1^{d}$ & $136 \pm 4^{\mathrm{d}}$ & $14.2 \pm 0.4^{\mathrm{d}}$ \\
\hline $\mathrm{S}_{\mathrm{CF} 75}+\mathrm{F}_{\mathrm{CF} 25}$ & $1.69 \pm 0.03^{c}$ & $0.76 \pm 0.02^{\mathrm{c}}$ & $13.4 \pm 0.4^{\mathrm{c}}$ & $64.5 \pm 2.3^{c}$ & $163 \pm 5^{c}$ & $18.0 \pm 0.5^{\mathrm{c}}$ \\
\hline $\mathrm{S}_{\mathrm{CF} 75}+\mathrm{F}_{\mathrm{CF} 25}+\mathrm{C}_{\mathrm{L}}$ & $2.17 \pm 0.04^{b}$ & $0.83 \pm 0.03^{b}$ & $15.9 \pm 0.4^{b}$ & $74.0 \pm 2.5^{b}$ & $183 \pm 7^{b}$ & $22.1 \pm 0.5^{b}$ \\
\hline $\mathrm{S}_{\mathrm{CF} 75}+\mathrm{F}_{\mathrm{CF} 25}+\mathrm{C}_{\mathrm{L}} \mathrm{B}$ & $2.65 \pm 0.05^{\mathrm{a}}$ & $0.89 \pm 0.03^{a}$ & $18.5 \pm 0.4^{\mathrm{a}}$ & $82.3 \pm 2.9^{a}$ & $205 \pm 7^{a}$ & $26.4 \pm 0.6^{\mathrm{a}}$ \\
\hline
\end{tabular}


Table 6. Cont.

\begin{tabular}{|c|c|c|c|c|c|c|}
\hline \multirow{2}{*}{ Source of Variation } & \multicolumn{3}{|c|}{ Photosynthetic Parameters } & \multicolumn{3}{|c|}{$\begin{array}{c}\text { Leaf Relative Water Content (RWC) and } \\
\text { Osmoprotectant Contents }\end{array}$} \\
\hline & $\begin{array}{l}\text { TChl Content } \\
\left(\mathrm{g} \mathrm{kg}^{-1} \mathrm{FW}\right)\end{array}$ & Fv/Fm & PI (\%) & RWC (\%) & $\begin{array}{c}\text { Proline } \\
\left(\mu g^{-1} \mathrm{DW}\right)\end{array}$ & $\begin{array}{c}\text { TS Sugars } \\
\left(\mathrm{mg} \mathrm{g}^{-1} \mathrm{DW}\right)\end{array}$ \\
\hline $\mathrm{S} \times \mathrm{F}$ & $* *$ & * & * & * & $* *$ & ** \\
\hline $2019 \times S_{C F 100}$ & $1.12 \pm 0.02 \mathrm{e}^{\mathrm{e}}$ & $0.68 \pm 0.011^{\mathrm{e}}$ & $10.4 \pm 0.2^{f}$ & $52.4 \pm 1.9^{\mathrm{d}}$ & $125 \pm 3^{d}$ & $12.4 \pm 0.3^{\mathrm{e}}$ \\
\hline $2019 \times \mathrm{S}_{\mathrm{CF} 75}+\mathrm{F}_{\mathrm{CF} 25}$ & $1.52 \pm 0.02^{\mathrm{d}}$ & $0.73 \pm 0.02^{\mathrm{d}}$ & $12.4 \pm 0.3^{\mathrm{e}}$ & $60.2 \pm 2.1^{\mathrm{c}}$ & $148 \pm 4^{\mathrm{d}}$ & $15.8 \pm 0.4^{\mathrm{d}}$ \\
\hline $2019 \times \mathrm{S}_{\mathrm{CF} 75}+\mathrm{F}_{\mathrm{CF} 25}+\mathrm{C}_{\mathrm{L}}$ & $1.97 \pm 0.03^{c}$ & $0.79 \pm 0.02^{\mathrm{c}}$ & $14.9 \pm 0.3^{\mathrm{c}}$ & $69.6 \pm 2.4^{b}$ & $176 \pm 6^{\mathrm{c}}$ & $19.6 \pm 0.4^{\mathrm{c}}$ \\
\hline $2019 \times \mathrm{S}_{\mathrm{CF} 75}+\mathrm{F}_{\mathrm{CF} 25}+\mathrm{C}_{\mathrm{L}} \mathrm{B}$ & $2.44 \pm 0.04^{\mathrm{b}}$ & $0.86 \pm 0.02^{b}$ & $17.2 \pm 0.4^{b}$ & $77.8 \pm 2.9^{a}$ & $192 \pm 6^{b c}$ & $24.0 \pm 0.5^{b}$ \\
\hline $2020 \times S_{\text {CF100 }}$ & $1.48 \pm 0.03^{\mathrm{de}}$ & $0.74 \pm 0.02^{\mathrm{d}}$ & $12.6 \pm 0.3^{\text {de }}$ & $61.0 \pm 2.2^{c}$ & $147 \pm 5^{\mathrm{d}}$ & $16.0 \pm 0.4^{\mathrm{d}}$ \\
\hline $2020 \times \mathrm{S}_{\mathrm{CF} 75}+\mathrm{F}_{\mathrm{CF} 25}$ & $1.86 \pm 0.03^{\mathrm{cd}}$ & $0.79 \pm 0.02^{c}$ & $14.4 \pm 0.4^{\mathrm{cd}}$ & $68.8 \pm 2.5^{b}$ & $178 \pm 5^{c}$ & $20.2 \pm 0.5^{c}$ \\
\hline $2020 \times \mathrm{S}_{\mathrm{CF} 75}+\mathrm{F}_{\mathrm{CF} 25}+\mathrm{C}_{\mathrm{L}}$ & $2.37 \pm 0.04^{b}$ & $0.87 \pm 0.03^{b}$ & $16.9 \pm 0.4^{b}$ & $78.4 \pm 2.6^{\mathrm{a}}$ & $196 \pm 7^{\mathrm{ab}}$ & $24.6 \pm 0.5^{b}$ \\
\hline $2020 \times \mathrm{S}_{\mathrm{CF} 75}+\mathrm{F}_{\mathrm{CF} 25}+\mathrm{C}_{\mathrm{L}} \mathrm{B}$ & $2.86 \pm 0.05^{\mathrm{a}}$ & $0.92 \pm 0.03^{\mathrm{a}}$ & $19.8 \pm 0.4^{\mathrm{a}}$ & $86.8 \pm 2.8^{a}$ & $218 \pm 7^{\mathrm{a}}$ & $28.8 \pm 0.6^{\mathrm{a}}$ \\
\hline
\end{tabular}

${ }^{* *}$ and ${ }^{*}$ indicate, respectively, differences at $p \leq 0.05$ and $p \leq 0.01$ probability level, respectively. Mean values with different letters in each column are significant $(p \leq 0.05) . \mathrm{TChl}=$ total chlorophylls, $\mathrm{Fv} / \mathrm{Fm}=$ chlorophyll fluorescence, $\mathrm{PI}=$ performance index, $\mathrm{S}_{\mathrm{CF} 100}=$ chemical fertilizers applied to the soil at full recommended NPK doses, $\mathrm{S}_{\mathrm{CF} 75}=$ chemical fertilizers applied to the soil at $75 \%$ of recommended NPK doses, $\mathrm{F}_{\mathrm{CF} 25}=$ chemical fertilizers applied as foliar sprays at $25 \%$ of recommended NPK doses, $\mathrm{C}_{\mathrm{L}}=1.2 \mathrm{~kg}$ leguminous compost $\mathrm{m}^{-2}$, and $\mathrm{C}_{\mathrm{L}} \mathrm{B}$ = biocompost; leguminous compost $\left(1.2 \mathrm{~kg} \mathrm{~m}^{-2}\right)$ containing bacteria.

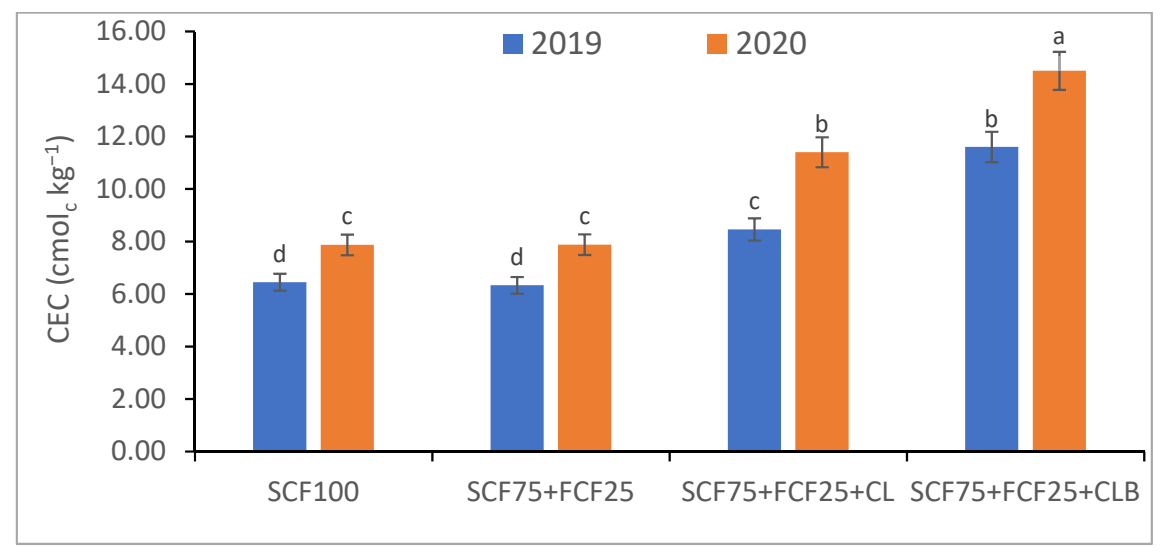

Figure 1. Response of cation exchange capacity to different fertilization strategies applied in two seasons (2019 and 2020) to sandy soil. Abbreviations of fertilization treatments are explained in Table 1. Bars with a different letter indicate significant difference between treatments at $p \leq 0.05$.

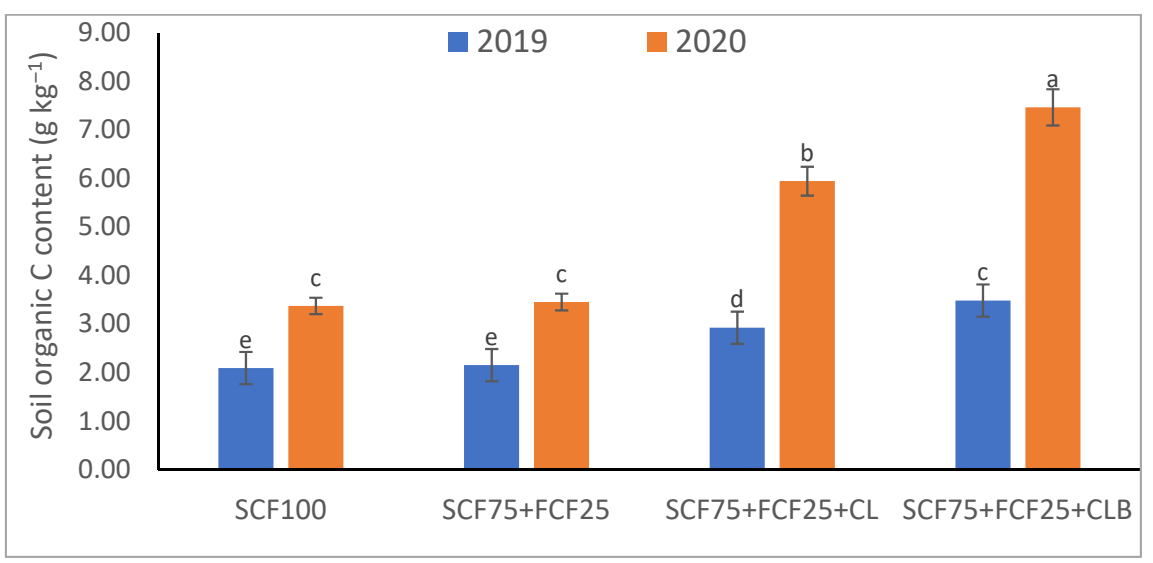

Figure 2. Response of soil organic $C$ content to different fertilization strategies applied in two seasons (2019 and 2020) to sandy soil. Abbreviations of fertilization treatments are explained in Table 1. Bars with a different letter indicate significant difference between treatments at $p \leq 0.05$. 


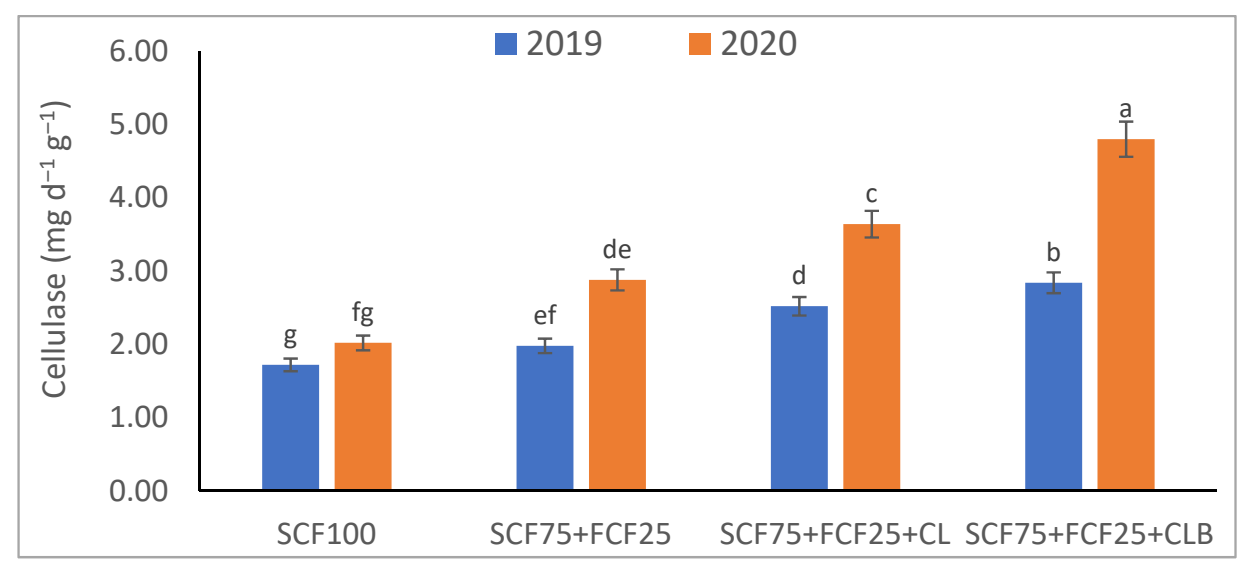

Figure 3. Response of soil enzyme activities (cellulase) to different fertilization strategies applied in two seasons (2019 and 2020) to sandy soil. Abbreviations of fertilization treatments are explained in Table 1. Bars with a different letter indicate significant difference between treatments at $p \leq 0.05$.

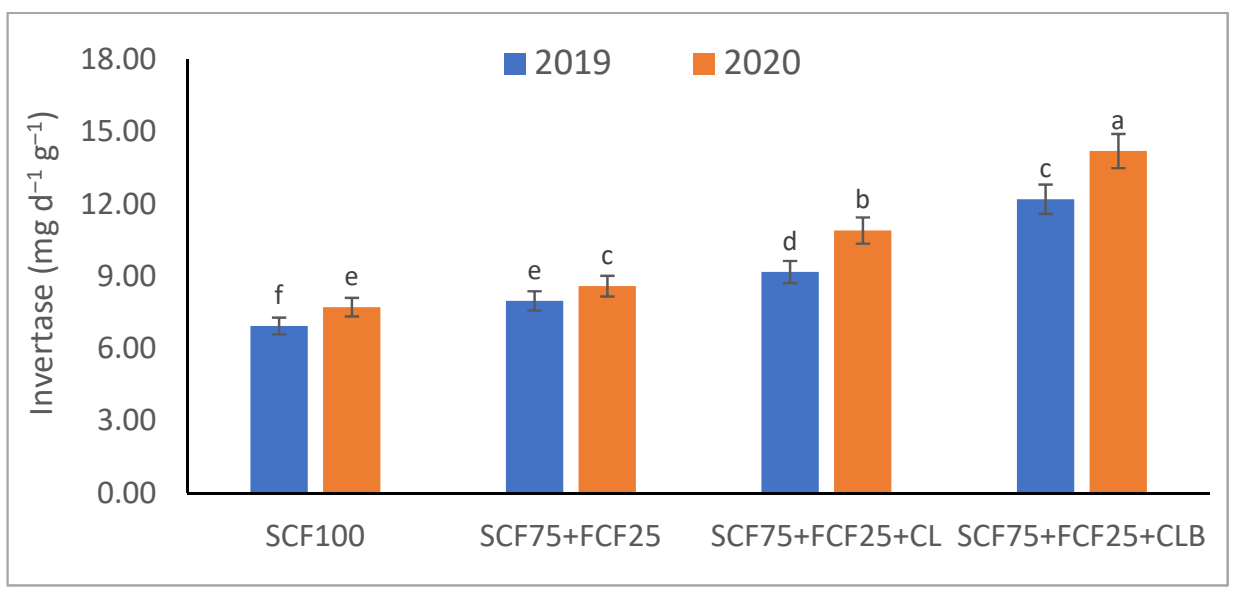

Figure 4. Response of soil enzyme activities (invertase) to different fertilization strategies applied in two seasons (2019 and 2020) to sandy soil. Abbreviations of fertilization treatments are explained in Table 1. Bars with a different letter indicate significant difference between treatments at $p \leq 0.05$.

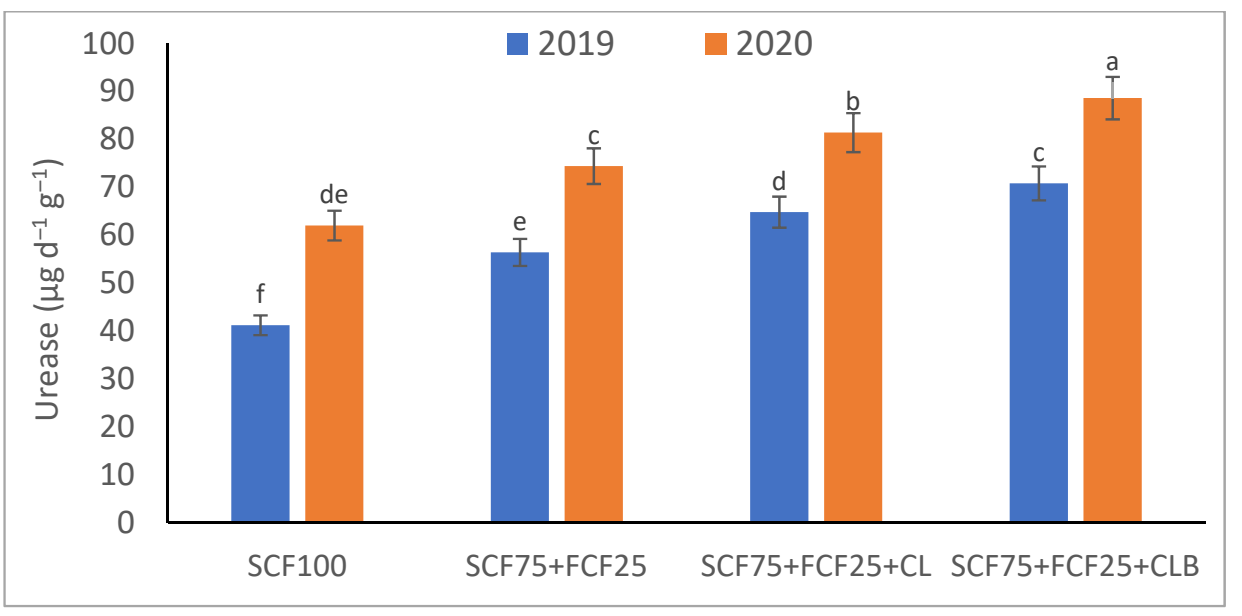

Figure 5. Response of soil enzyme activities (urease) to different fertilization strategies applied in two seasons (2019 and 2020) to sandy soil. Abbreviations of fertilization treatments are explained in Table 1. Bars with a different letter indicate significant difference between treatments at $p \leq 0.05$. 


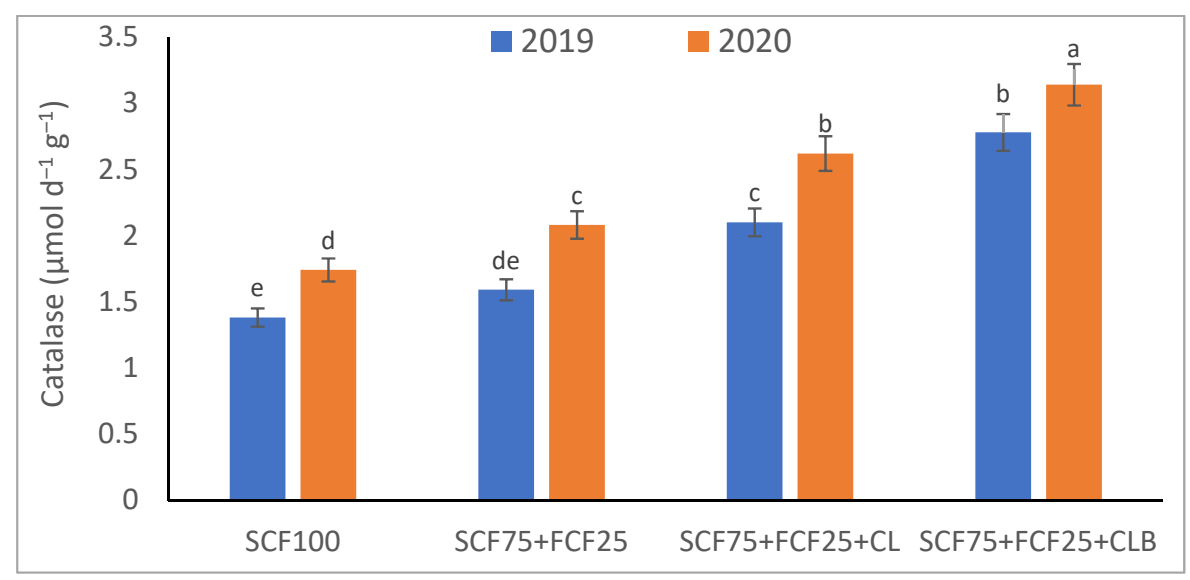

Figure 6. Response of soil enzyme activities (catalase) to different fertilization strategies applied in two seasons (2019 and 2020) to sandy soil. Abbreviations of fertilization treatments are explained in Table 1. Bars with a different letter indicate significant difference between treatments at $p \leq 0.05$.

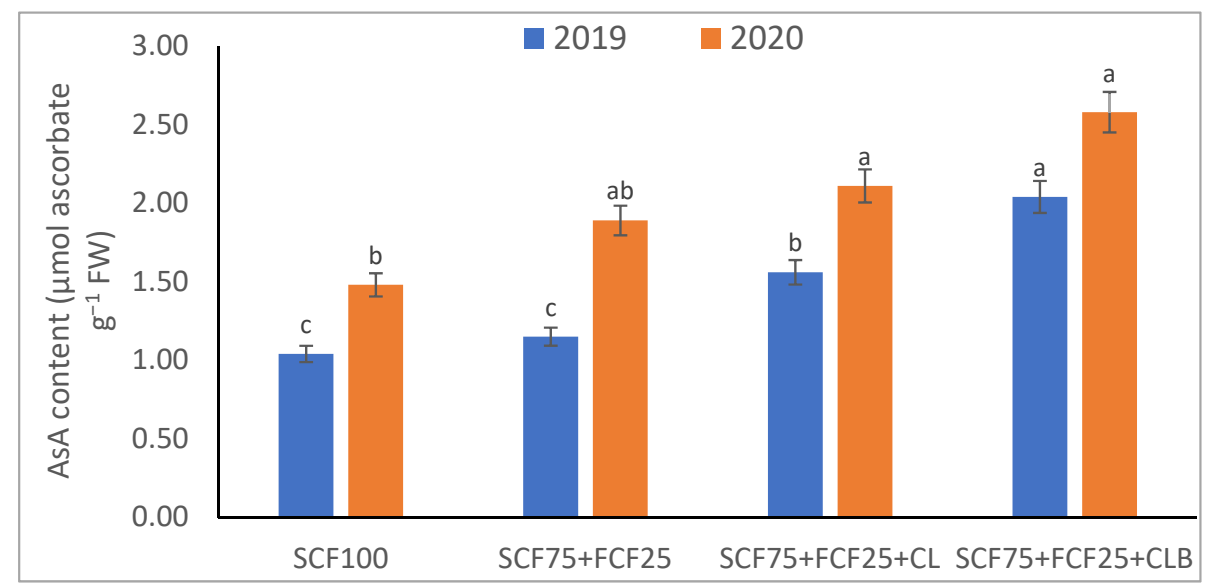

Figure 7. Response of leaf nonenzymatic antioxidants contents (ascorbic acid-AsA) of Phaseolus vulgaris plants to different fertilization strategies applied in two seasons (2019 and 2020) to sandy soil. Abbreviations of fertilization treatments are explained in Table 1. Bars with a different letter indicate significant difference between treatments at $p \leq 0.05$.

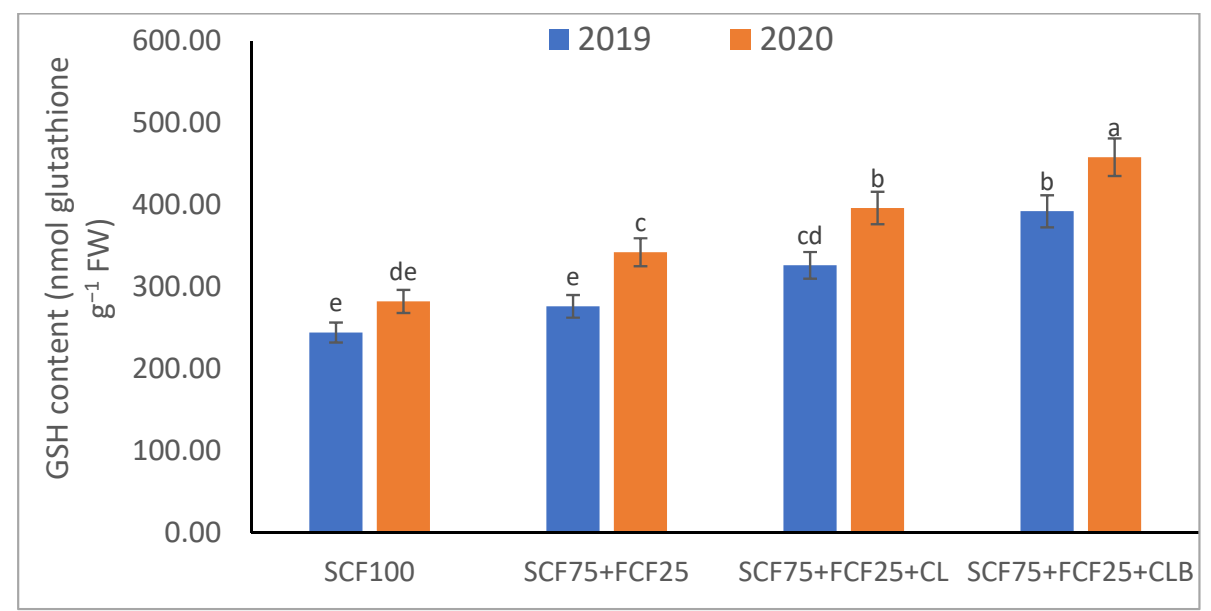

Figure 8. Response of leaf nonenzymatic antioxidants contents (glutathione-GSH) of Phaseolus vulgaris plants to different fertilization strategies applied in two seasons (2019 and 2020) to sandy soil. Abbreviations of fertilization treatments are explained in Table 1. Bars with a different letter indicate significant difference between treatments at $p \leq 0.05$. 


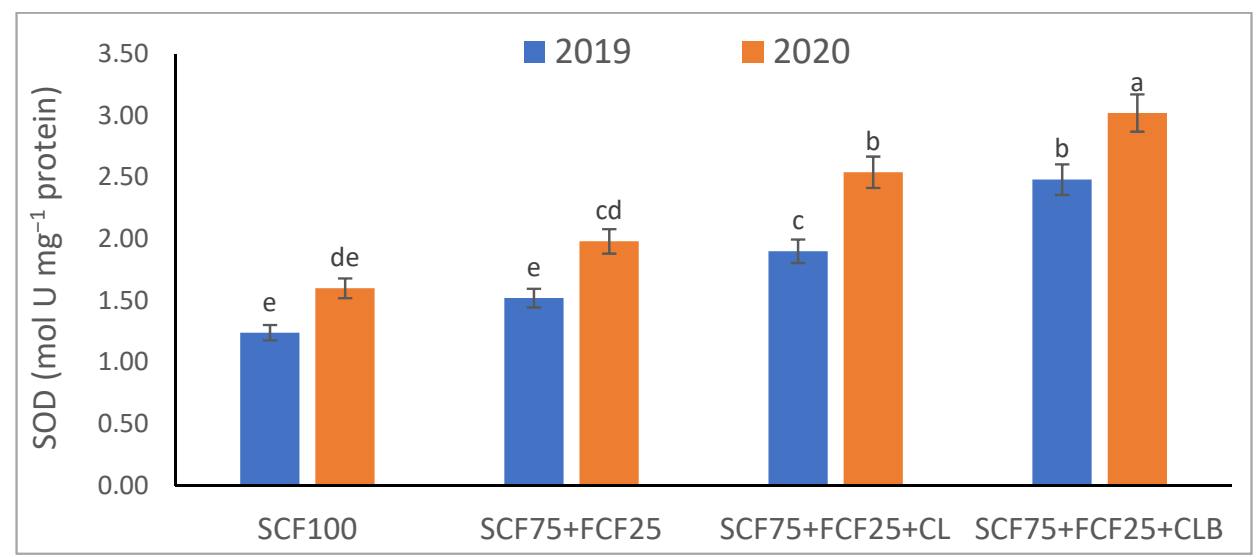

Figure 9. Response of leaf enzyme activity (superoxide dismutase-SOD) of Phaseolus vulgaris plants to different fertilization strategies applied in two seasons (2019 and 2020) to sandy soil. Abbreviations of fertilization treatments are explained in Table 1. Bars with a different letter indicate significant difference between treatments at $p \leq 0.05$.

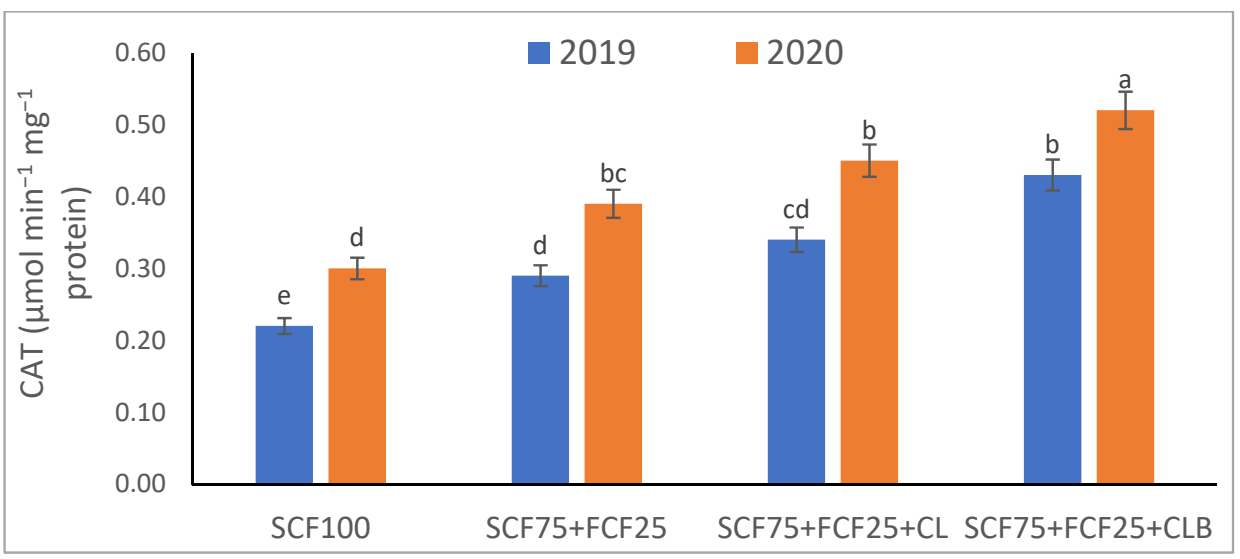

Figure 10. Response of leaf enzyme activity (catalase-CAT) of Phaseolus vulgaris plants to different fertilization strategies applied in two seasons (2019 and 2020) to sandy soil. Abbreviations of fertilization treatments are explained in Table 1. Bars with a different letter indicate significant difference between treatments at $p \leq 0.05$.

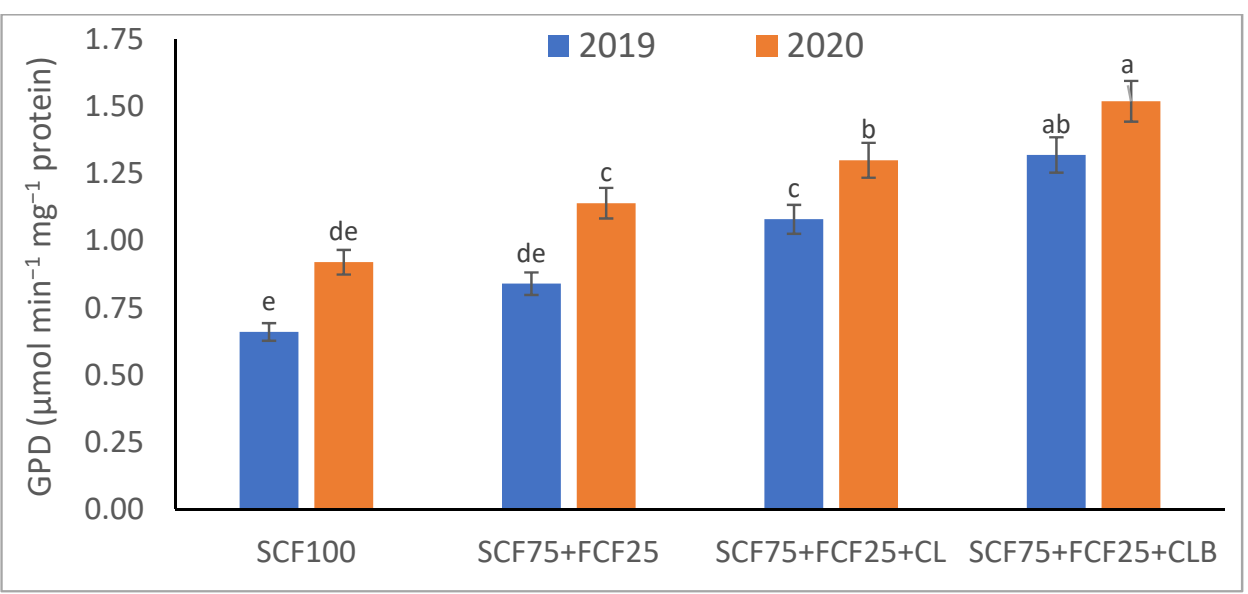

Figure 11. Response of leaf enzyme activity (guaiacol peroxidase-GPD) of Phaseolus vulgaris plants to different fertilization strategies applied in two seasons (2019 and 2020) to sandy soil. Abbreviations of fertilization treatments are explained in Table 1. Bars with a different letter indicate significant difference between treatments at $p \leq 0.05$. 


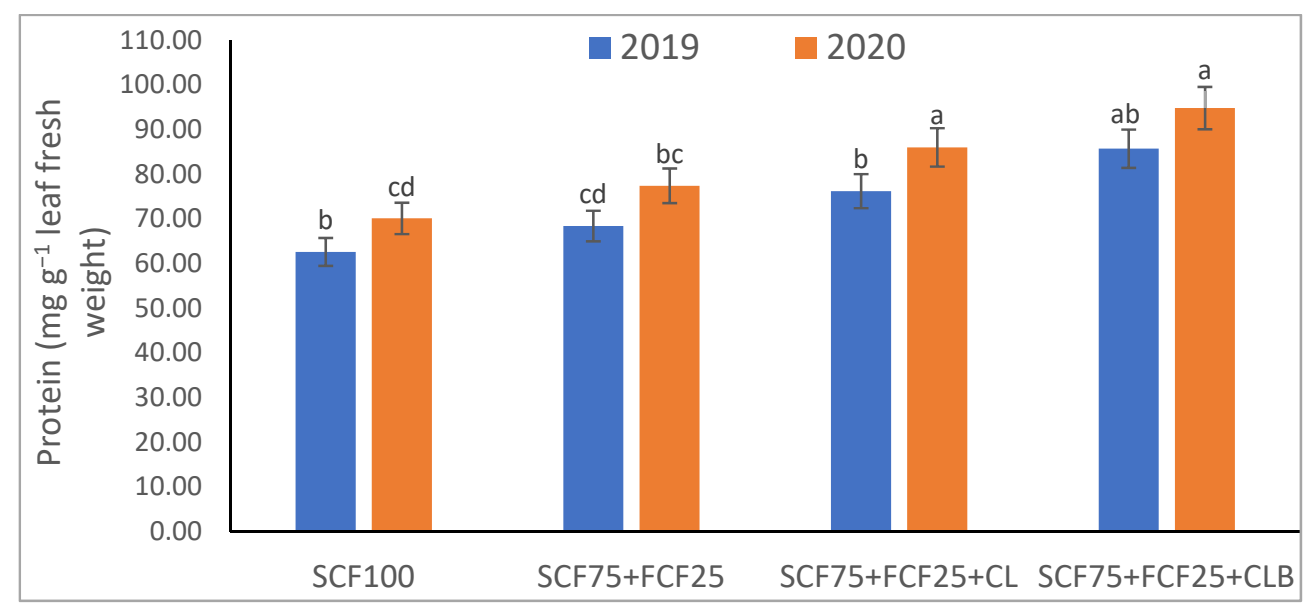

Figure 12. Response of leaf enzyme activity (protein) of Phaseolus vulgaris plants to different fertilization strategies applied in two seasons (2019 and 2020) to sandy soil. Abbreviations of fertilization treatments are explained in Table 1. Bars with a different letter indicate significant difference between treatments at $p \leq 0.05$.

In the 2020 growing season, there were significant $(p \leq 0.05)$ increases in available $\mathrm{N}$ at $18.5 \%, \mathrm{~K}$ at $21.3 \%$, Fe at $31.9 \%$, Mn at $45.7 \%$, and $\mathrm{Zn}$ at $42.3 \%$ compared to those obtained in the 2019 growing season (Table 3). The data in Figures 1-6 display that the cation exchange capacity (CEC), organic $C$ content, cellulose, invertase, urease, and catalase activities of the tested soil were significantly increased in the 2020 growing season compared to those achieved in the 2019 season. As shown in Table 4, shoot fresh and dry weights, and green pods and dry seed weights of Phaseolus vulgaris plants were significantly $(p \leq 0.05)$ increased by $20.8,15.6,14.5$, and $28.4 \%$, respectively, in the 2020 season compared to those gained in the 2019 season. The plant nutrient contents represented the same behavior of the soil nutrient contents, since $\mathrm{N}, \mathrm{P}, \mathrm{K}, \mathrm{Fe}, \mathrm{Mn}$, and $\mathrm{Zn}$ contents were significantly $(p \leq 0.05)$ increased by $19.9,25.4,15.8,17.5,23.9$, and $19.8 \%$ in the 2020 growing season compared to those collected in the 2019 season (Table 5). The data in Table 6 and Figures 7-12 revealed that the physiobiochemical attributes (e.g., total chlorophyll content, Fv /Fm, PSII performance index, leaf relative water content, proline content, total soluble sugar content, ascorbate content, glutathione content, protein content, and leaf enzymatic activity of guaiacol peroxidase, catalase, and superoxide dismutase) of Phaseolus vulgaris plants were significantly elevated in the 2020 season compared to those determined in the 2019 season. The total chlorophyll content, Fv / Fm, PSII performance index, leaf relative water content, proline content, total soluble sugar contents were increased by 21.6, 7.8, 16.1, 13.5, 15.6, and $24.4 \%$, respectively. Besides this, the contents of ascorbate, glutathione, and protein, as well as the activities of guaiacol peroxidase, catalase, and superoxide dismutase, were increased by $39.3,19.4,27.9,31.3,24.5$, and 12.2 , respectively.

\subsection{Response of Soil Chemical Properties and Plant Performances to Different Fertilization Strategies}

Some fertilization strategies (sub-main factor) were applied to the nutrient-deficient virgin sandy soil tested in this study, and they positively influenced the soil chemical properties, soil and plant contents of essential nutrients and activities of various enzymes, plant growth and productivity, and plant physiobiochemistry (Tables 3-6 and Figures 1-12).

All blended fertilization strategies used in this study significantly increased the chemical properties of the tested soil, soil and plant contents of essential nutrients and activities of various enzymes, plant growth and productivity, and the plant physiobiochemistry, excluding some exceptions recorded by $\mathrm{S}_{\mathrm{CF} 75}+\mathrm{F}_{\mathrm{CF} 25}$ treatment compared to control $\left(\mathrm{S}_{\mathrm{CF} 100}=\right.$ chemical fertilizers applied to the soil at full recommended NPK doses). Among all fertilization strategies, the $\mathrm{S}_{\mathrm{CF} 75}+\mathrm{F}_{\mathrm{CF} 25}+\mathrm{C}_{\mathrm{L}} \mathrm{B}$ was the best treatment. It conferred $(p \leq 0.05)$ significant increases in soil content of available N, Mn, and Zn by 56.7, 61.3, and 
$50.9 \%$, respectively (Table 3), and soil cellulase and urease activities by 80.1 and $54.5 \%$, respectively (Figures 3 and 5), compared to control. Besides this, it awarded highly significant $(p \leq 0.01)$ increases in soil content of available $\mathrm{P}, \mathrm{K}$, and Fe by $84.7,58.9$, and $82.6 \%$, respectively (Table 3), and soil CEC, organic $\mathrm{C}$ content, and invertase and catalase activities by $83.0,100.4,104.3$, and $89.7 \%$, respectively (Figures 4 and 6 ), compared to control. For Phaseolus vulgaris plant, the best treatment $\left(\mathrm{S}_{\mathrm{CF75}}+\mathrm{F}_{\mathrm{CF} 25}+\mathrm{C}_{\mathrm{L}} \mathrm{B}\right)$ also conferred highly significant $(p \leq 0.01)$ increases in shoot fresh weight and dry seed weight by 70.6 and $109.6 \%$, respectively (Table 4), contents of $\mathrm{P}, \mathrm{Mn}, \mathrm{Zn}$, total chlorophylls, proline, soluble sugars, and ascorbate by $98.6,83.3,76.0,103.8,50.7,85.9$, and 83.3\%, respectively (Tables 5 and 6 ; Figure 7), and activities of superoxide dismutase, catalase, and guaiacol peroxidase by 93.7, 84.6, and 79.7\%, respectively (Figures 9-11), compared to control. It contributed significant $(p \leq 0.05)$ increases in shoot dry weight and green pods weight by 51.3 and $56.9 \%$, respectively (Table 4), contents of $\mathrm{N}, \mathrm{K}, \mathrm{Fe}$, glutathione, and protein by $73.4,68.0$, 71.0, 61.6, and 36.0\%, respectively (Table 5; Figures 8 and 12), and in Fv/Fm, performance index, and relative water content by $25.4,60.9$, and $45.1 \%$, respectively (Table 6), compared to control $\left(\mathrm{S}_{\mathrm{CF} 100}\right)$.

\subsection{Response of Soil Chemical Properties and Plant Performances to the Interaction between Growing Season and Different Fertilization Strategies}

The interaction between growing season and fertilization strategies and their influences on the chemical properties of the tested soil, soil and plant contents of essential nutrients and activities of various enzymes, plant growth and productivity, and plant physiobiochemistry under nutrient-deficient virgin sandy soil conditions are shown in Tables 3-6 and Figures 1-12.

Among all interactions, the application of $2019 \times \mathrm{S}_{\mathrm{CF} 100}$ (control) or $2019 \times \mathrm{S}_{\mathrm{CF} 75}+$ $\mathrm{F}_{\mathrm{CF} 25}$ collected the lowest findings of all determined soil and plant parameters. However, $2020 \times \mathrm{S}_{\mathrm{CF} 75}+\mathrm{F}_{\mathrm{CF} 25}+\mathrm{C}_{\mathrm{L}} \mathrm{B}$ was the best interaction treatment, which conferred significant $(p \leq 0.05)$ or highly significant $(p \leq 0.01)$ increases in all tested soil and plant parameters. It conferred $(p \leq 0.05)$ significant increases in soil content of available N, Mn, and Zn by 82.0, 149.1 , and $120.7 \%$, respectively (Table 3), and soil cellulase and urease activities by 104.6 and $115.0 \%$, respectively (Figures 3 and 5), compared to control. Besides, it contributed highly significant $(p \leq 0.01)$ increases in soil content of available $\mathrm{P}, \mathrm{K}$, and Fe by 131.7, 91.0, and $131.5 \%$, respectively (Table 3), and soil CEC, organic C content, and invertase and catalase activities by $124.8,256.9,179.1$, and $127.5 \%$, respectively (Figures 1-6), compared to control. For Phaseolus vulgaris plant, the best interaction treatment $\left(2020 \times \mathrm{S}_{\mathrm{CF} 75}+\mathrm{F}_{\mathrm{CF} 25}+\mathrm{C}_{\mathrm{L}} \mathrm{B}\right)$ also conferred highly significant $(p \leq 0.01)$ increases in shoot fresh weight and dry seed weight by 102.1 and $165.2 \%$, respectively (Table 4 ), contents of $\mathrm{P}, \mathrm{Mn}, \mathrm{Zn}$, total chlorophylls, proline, soluble sugars, and ascorbate by 159.0, 121.9, 109.0, 155.4, 74.4, 132.3, and 124.3\%, respectively (Tables 5 and 6; Figure 7), and activities of superoxide dismutase, catalase, and guaiacol peroxidase by $143.5,136.4$, and $130.3 \%$, respectively (Figures $9-11$ ), compared to control. It contributed significant $(p \leq 0.05)$ increases in shoot dry weight and green pods weight by 75.4 and $82.7 \%$, respectively (Table 4 ), contents of $\mathrm{N}, \mathrm{K}, \mathrm{Fe}$, glutathione, and protein by $103.9,94.4,100.0,87.7$, and $51.4 \%$, respectively (Table 5; Figures 8 and 12), and in $\mathrm{Fv} / \mathrm{Fm}$, performance index, and relative water content by $35.3,90.4$, and $65.6 \%$, respectively (Table 6), compared to control $\left(\mathrm{S}_{\mathrm{CF} 100}\right)$.

\section{Discussion}

Climate change, as one of the major challenges facing the world at present, has negatively affected agricultural lands. Furthermore, future changes in climatic events are expected to worsen [58], particularly in arid and semi-arid regions [59-63]. As a consequence, soils have lost much of their fertility, threatening the food security of the world [58,64-70]. Accordingly, to maintain sustainable agricultural development, virgin (unused) lands that can be cultivated with more organic and less chemical fertilization, are being, or should be, used to minimize pollutants to produce clean agricultural products free of pollution [71]. 
Sandy soils suffer from high permeability coupled with poor water retention, which results in the loss of many important nutrients due to poor ability to retain many important nutrients, besides, the competition of cations, and consequently the rapid leaching [1,72-74]. Besides, if such soil was virgin, its suffering would have been greater. Therefore, it is necessary to improve the weaknesses of this soil by nourishing it with organic fertilizers, especially biocomposts, because they enhance microbial decomposition leading to humification of organic matter [75] in favor of the soil and plants growing on it.

The virgin sandy soil used in this study had very low water and nutrient holding capacity and a poor cation exchange capacity (CEC), making it impossible to rely on adding nitrogen, phosphorus, and potassium (NPK) fertilizers only, otherwise, these fertilizers are rapidly lost. Therefore, to minimize fertilizer nutrient loss, it is appropriate to add a portion of the recommended NPK fertilizer (about 25\%) to the plant as a foliar spray in combination with adding another portion (about $75 \%$ ) to the soil with the addition of an appropriate amount of organic $\left(\mathrm{S}_{\mathrm{CF} 75}+\mathrm{F}_{\mathrm{CF} 25}+\mathrm{C}_{\mathrm{L}}\right)$ or bio-organic fertilizer $\left(\mathrm{S}_{\mathrm{CF} 75}+\mathrm{F}_{\mathrm{CF} 25}+\mathrm{C}_{\mathrm{L}} \mathrm{B}\right)$ as a required strategy for the sustainability and productivity of plants growing on the tested soil. From this point of view, the $\mathrm{S}_{\mathrm{CF75}}+\mathrm{F}_{\mathrm{CF} 25}$ treatment went beyond the control treatment and significantly increased soil physicochemical properties (Tables 2 and 3; Figures 1-6) and Phaseolus vulgaris plant growth, productivity, and physiobiochemical attributes (Tables 4-6; Figures 7-12). Enrichment of the $\mathrm{S}_{\mathrm{CF75}}+\mathrm{F}_{\mathrm{CF} 25}$ treatment with leguminous compost $\left(\mathrm{C}_{\mathrm{L}}\right)$ at $1.2 \mathrm{~kg}$ per $\mathrm{m}^{2}\left(\mathrm{~S}_{\mathrm{CF} 75}+\mathrm{F}_{\mathrm{CF} 25}+\mathrm{C}_{\mathrm{L}}\right)$ increased the treatment efficiency, as it exceeded the $\mathrm{S}_{\mathrm{CF} 75}+\mathrm{F}_{\mathrm{CF} 25}$ treatment, resulting in a significant increase in the tested soil and plant attributes (Tables 3-6; Figures 1-12). Moreover, enrichment of the $C_{\mathbf{L}}$ in the $S_{\mathrm{CF} 75}+\mathrm{F}_{\mathrm{CF} 25}+$ $\mathrm{C}_{\mathrm{L}}$ treatment with bacteria (Bacillus subtilis) further increased the treatment efficiency, as the $\mathrm{S}_{\mathrm{CF75}}+\mathrm{F}_{\mathrm{CF} 25}+\mathrm{C}_{\mathrm{L}} \mathrm{B}$ treatment greatly exceeded the $\mathrm{S}_{\mathrm{CF75}}+\mathrm{F}_{\mathrm{CF} 25}+\mathrm{C}_{\mathrm{L}}$ treatment, resulting in a further significant improvement in soil and plant attributes (Tables 2-6; Figures 1-12).

Therefore, from our findings of the tested virgin sandy soil and Phaseolus vulgaris plant, the $\mathrm{S}_{\mathrm{CF} 75}+\mathrm{F}_{\mathrm{CF} 25}+\mathrm{C}_{\mathrm{L}} \mathrm{B}$ treatment was the best, with the best improvement results for the soil texture, $\mathrm{pH}, \mathrm{EC}_{\mathrm{e}}$, and $\mathrm{CaCO}_{3}$ content in both the 2019 and 2020 seasons (Tables 2-6; Figures 1-12). These improvements were attributed to the leguminous biocompost $\left(C_{L} B\right)$, which provided soil characteristics, in addition to the NPK nutrients that the plant acquired through two pathways: roots and leaves. In addition, compared to all treatments, including the control, the $\mathrm{S}_{\mathrm{CF} 75}+\mathrm{F}_{\mathrm{CF} 25}+\mathrm{C}_{\mathrm{L}} \mathrm{B}$ treatment significantly increased $\mathrm{CEC}$, soil organic carbon (C) content, soil nutrient (N, P, K, Fe, Mn, and Zn) contents, and soil enzymes (e.g., cellulase, invertase, urease, and catalase) activities (Table 3 and Figures 1-6).

Soil $\mathrm{pH}$ was decreased with soil supplementation using $\mathrm{C}_{\mathrm{L}} \mathrm{B}$, which could be attributed to the increase in organic $C$ content and soil enzyme activities (Figures 1-6), which led to the decomposition of the added organic matter (in $\mathrm{C}_{\mathbf{L}} \mathrm{B}$ ). Besides, organic acids and phytohormones (e.g., indole acetic acid and cytokinins) resulting from the activity of bacteria added to the compost, leading to an increase in biological activity [33,76-78]. As obtained in this study (Table 2), a decrease in $\mathrm{pH}$ is reported with the combined use of biocompost and inorganic fertilizer [79]. This positive result is attributed to the production of organic acids due to biocompost decomposition followed by an increase in the salt content of the soil due to mineralization, which increases the EC of the soil. Sinha et al. [79] also reported that soil $\mathrm{pH}$ decreased while EC increased, due to biocompost application, which also had a significant influence on soil organic $C$ content and available nutrients (e.g., N, P, K, and S).

Furthermore, supplementing the soil with $C_{L} B$ enhanced the microbial decomposition that leads to an increase in soil organic matter and thus CEC (Figures 1-6). Buragohain et al. [75] found that the biocompost generates a large number of different bacteria that cause an increase in soil organic matter, which leads to improved crop growth. This subsequently results in increased exudations of plant roots and return of post-harvest residues, thus contributing to the organic content of the soil. They also documented that the observed high contribution of biocompost to soil organic $C$ indicates more rebellious forms of soil organic $C$, thus resulting in enhanced soil $C$ stabilization. Soil organic $C$ 
elevation is also observed following the application of biofertilizers (Bacillus megatherium and Bacillus mucilaginous), as per $\mathrm{Wu}$ et al. [80]. Increase of CEC after application of compost [21], and in particular biocompost, is an indication of a high accumulation of soil organic $\mathrm{C}$ pool along with low inorganic $\mathrm{P}$ and $\mathrm{N}$. The decreased inorganic $\mathrm{P}$ and $\mathrm{N}$ following the application of biocompost contribute to building a soil environment where a desirable microbial composition is created to stabilize the soil organic carbon pool [75]. They also reported that the quality of organic matter and fertility of the soil is improved due to the increased humic/fulvic acid ratio due to the increased rate of humification, which helps to raise the stability of organic $C$ as a result of biocompost supplementation to the soil [81].

The buildup of available nutrients in nutrient-deficient soils, such as the soil tested in this study, can be attributed to the increased microbial proliferation due to the addition of organic manures, especially biocompost, which helps in mineralization as well as solubilization of native nutrients by complexation of nutrients by humic and fulvic acid contained in biocompost [79]. The results of this study indicated that the application of NPK fertilizers only was not effective in maintaining soil fertility (Tables 2 and 3; Figures 1-6). The nutrients available in the soil and soil organic $C$ were preserved in the treatment containing biocompost. It has been reported by Sinha et al. [79] that soil fertility-contributing bulk density and pore spaces are improved effectively due to the accumulation of organic $C$ content of the biocompost-treated soil. The beneficial influences of biocompost in improving the soil's physical and chemical properties may be attributed to the improvement in the organic matter status of the soil treated with biocompost, which leads to the buildup of soil fertility for sustainable production of Phaseolus vulgaris. Sinha et al. [79] also explained that the use of biocompost greatly increases the soil microbial population, which utilizes the accumulated organic $C$ as a source of energy, nutrients, and nourishment, which leads to the spread of microorganisms in the soil. This improved microbial community and activity due to the accumulation of organic matter by the application of biocompost help maintain soil fertility and productivity due to the faster decomposition rate and smooth mineralization of organic materials [79].

As shown in the data of this study, the combined use of biocompost and inorganic fertilizers (applied to both soil and plant leaves) effectively increased the uptake of nutrients, which reflected positively on the higher yield (Tables 4 and 5). Managing nutrients through organic and inorganic sources leads to more nutrient uptake. As of the effectively increased parameters due to the combined use of $C_{L} B$ and inorganic fertilizers, the soil organic $C$ content and availability of nutrients (e.g., N, P, K, Fe, Mn, and $\mathrm{Zn}$ ) in the tested virgin sandy soil may be attributed to the increased content of organic $C$ and nutrients in the $C_{L} B$ and the beneficial advantages of inorganic nutrients. These nutrients are released from the compost into the soil through bacterial decomposition [82] present in $C_{L} B$, which is activated by the added compost. Manirakiza and Şeker [77] reported increased contents of soil nutrients and organic $C$ which could be attributed to the compost's richness in organic $C$ and various nutrients. The release of nutrients from compost into the soil via the mineralization process can elucidate this result $[77,83]$.

Compared to all other treatments, including the control, the $\mathrm{S}_{\mathrm{CF} 75}+\mathrm{F}_{\mathrm{CF} 25}+\mathrm{C}_{\mathrm{L}} \mathrm{B}$ treatment significantly increased soil enzymes (e.g., cellulase, invertase, urease, and catalase) activities, especially after adding $C_{L} B$, indicating a potentially greater source of beneficial microbes. The increased activity of soil enzymes contributed to the release of more nutrients for microbial use [75]. Besides, the bacteria present in the $C_{L} B$ have desirable influences on increasing and stabilizing bacterial populations [84], which contributed along with the increased soil enzyme activities to repair limitations of the soil tested.

Macci et al. [85] and Buragohain et al. [75] demonstrated that compost and biocompost fertilizers increase soil quality due to enhancements of soil properties related to physicochemistry and biochemistry. This positive finding is confirmed by the results obtained for the tested virgin sandy soil, which reflected positively in the growth, productivity, and physiobiochemical indices of Phaseolus vulgaris plants (Tables 4-6; Figures 7-12). The 
increased availability of nutrients to the plants as a result of the improved physicochemical properties of the tested soil under supplementation of biocompost contributed to the increase in growth and physiobiochemical indices, and thus the productivity of $P$. vulgaris plants. As reported in [86], compost enriched with many bacterial strains (e.g., Bacillus, Rhizobium, Azotobacter, Azospirillum, Bradyrhizobium, Acetobacter, and Pseudomonas) has been reported to improve yields of different crops in some defective soils. Furthermore, it has been explained by Sinha et al. [79] that the immediate and rapid supply of nutrients through an inorganic source for plant growth and a steady supply of nutrients by organics, especially biocompost, throughout the growth period leads to an increase in plant yield due to integrated use of organic and inorganic nutrients. The biocompost releases nutrients after decomposition and mineralization that will increase the availability of nutrients at a later stage and improve physical, chemical, and biological properties of soil, resulting in improved soil fertility and absorption of nutrients by the plant. The integrated use of organic nutrient sources with inorganic fertilizers has been shown to increase the potential of organic fertilizers [79].

The growth and yield trend after two consecutive trial years of $P$. vulgaris plants showed that reducing $25 \%$ of the recommended NPK fertilizer dose of soil addition for use in foliar spraying along with the application of biocompost resulted in a progressive increase in the growth and yield of P. vulgaris plants (Table 4). Thus, the $\mathrm{S}_{\mathrm{CF75}}+\mathrm{F}_{\mathrm{CF} 25}+$ $C_{L} B$ treatment represented the highest sustainability for a period of two seasons (2019 and 2020). Allocating $25 \%$ of the NPK fertilizers to foliar spray conferred the opportunity to reduce the inorganic minerals added to the soil along with the use of $C_{L} B$ to conserve the soil while increasing its fertility (Tables 2 and 3; Figures 1-6). Besides, it increased the efficiency of the growing $P$. vulgaris plants in sandy soil to obtain all their nutritional needs, especially in the presence of $C_{L} B$ that facilitates nutrient uptake. Foliar spraying with NPK nutrients has become a concern of scientists for its dynamic application to increase plant growth and yield [17,87] (Tables 2-6; Figures 1-12).

As explained in [88], the increase in plant growth and productivity can be attributed to the positive influences of biocompost and its content of microorganisms in raising root surfaces, root distribution, water-use efficiency, and photosynthetic activity. These positive results directly affect the physiological processes and carbohydrate metabolism due to the high levels of nutrients and organic matter in the applied biocompost. Similarly, our data attributed the increases in growth and productivity of $P$. vulgaris plants to the application of $C_{L} B$, which contributed to improved phytonutrient contents (Table 3), photosynthetic efficiency (chlorophyll content, chlorophyll fluorescence, and PSII performance index), and relative cellular water content (Table 4), as well as the plant's antioxidant defense system; osmoprotectants (proline and sugars) and both enzymatic and nonenzymatic antioxidants (Table 6 and Figures 7-12).

The physicochemical properties and fertility of the virgin sandy soil, including the soil's organic $C$ content and organic acids, which were greatly improved by applying $C_{L} B$ (Tables 2 and 3; Figures 1-6), led to an increase in water retention in the soil [89], allowing P. vulgaris plants to absorb more water and nutrients, leading to increased relative cellular water content and different nutrient contents (Tables 4-6). Relative cellular water content indicates the water status in plants, reflecting the activity of plant metabolic processes, and is utilized as an indicator to distinguish between legumes with contrasting variations in drought tolerance [90]. The increased plant water and nutrient contents through the application of $\mathrm{S}_{\mathrm{CF} 75}+\mathrm{F}_{\mathrm{CF} 25}+\mathrm{C}_{\mathrm{L}} \mathrm{B}$ played crucial roles in improving the efficiency of photosynthesis and the antioxidant defense system (Table 4 and Figures 7-12), which need water as a medium for their reactions to take place $[22,91]$. The $S_{C F 75}+F_{C F 25}+C_{L} B$, including $\mathrm{C}_{\mathrm{L}} \mathrm{B}$, notably improved $P$. vulgaris plant water content due to the improved water uptake by roots and as a result of the accumulation of elemental $\mathrm{K}^{+}$, free proline, and soluble sugars as osmoprotectant compounds $[47,73]$. Furthermore, the accumulation of ascorbate and glutathione with the $\mathrm{S}_{\mathrm{CF} 75}+\mathrm{F}_{\mathrm{CF} 25}+\mathrm{C}_{\mathrm{L}} \mathrm{B}$ treatment contributed to the improved tissue water status and membrane integrity by reducing the activity of the 
reactive oxygen species (ROS) $[47,92,93]$. It has been explained that the accumulation of $\mathrm{K}^{+}$, free proline, and soluble sugars as osmolytes contributes to osmotic adjustment, which helps to maintain the cell turgor and stabilize the membranes by activation of antioxidant plant defense system [73,94-99]. This is also evident from improvement in antioxidant enzymes activities in this study (Figures 7-12), such as superoxide dismutase (SOD), catalase (CAT), and guaiacol peroxidase (GPOD), including leaf content of protein for the $\mathrm{S}_{\mathrm{CF} 75}+\mathrm{F}_{\mathrm{CF} 25}+\mathrm{C}_{\mathrm{L}} \mathrm{B}$ treatment in P. vulgaris plants grown under the tested conditions. Enhanced activities of assayed antioxidant enzymes with the $\mathrm{S}_{\mathrm{CF75}}+\mathrm{F}_{\mathrm{CF} 25}+\mathrm{C}_{\mathrm{L}} \mathrm{B}$ treatment have been also associated with improved photosynthetic activity and carbohydrates supply to growing sink [73], which contributed towards increased growth and yield of P. vulgaris plants. This increase in P. vulgaris plant performance with the $\mathrm{S}_{\mathrm{CF75}}+\mathrm{F}_{\mathrm{CF} 25}+\mathrm{C}_{\mathrm{L}} \mathrm{B}$ treatment is also associated with its influences on different physiological mechanisms and enzymes such as starch metabolism and glucose transport during photosynthesis and accumulation period [100]. All these findings were reflected, positively, in P. vulgaris plant growth and productivity (Table 4).

Soil application with $C_{L} B$ (compost + bacteria) can integrate conventional NPK fertilizers that should be applied to both soil and plant (foliar spray) in the cultivation of $P$. vulgaris and other crops using the defective sandy soils to improve plants' efficiency for good production and minimize contamination of farmland and agricultural products achieving agricultural sustainability.

All results of the 2020 season notably exceeded those of the 2019 season in terms of all the attributes examined for soil physicochemical properties and $P$. vulgaris plant growth, yield, and physiobiochemistry (Tables 2-6; Figures 1-12). This may be attributed to the residual effects of the first year's treatments, which provided the opportunity to liberate nutrients in excess amounts from the soil due to the elevated bacterial decomposition of the biocompost applied in the preceding season (2019), as well as the increased solubility of nutrients that contained in the biocompost.

\section{Conclusions}

The positive influences of leguminous biocompost in integration with reducing $25 \%$ of the recommended NPK fertilizers on soil fertility and physicochemical properties, as well as $P$. vulgaris plant growth, productivity, and physiobiochemical attributes, have been elucidated in a virgin sandy soil. Soil application with leguminous biocompost at a rate of $1.2 \mathrm{~kg}$ per $\mathrm{m}^{2}+75 \%$ of the recommended NPK fertilizer doses in integration with $25 \%$ of the recommended NPK fertilizer doses as a foliar spray for $P$. vulgaris plants resulted in significant improvements in soil fertility, physicochemical properties and plant physiobiochemical attributes, which were positively reflected in the plant growth and yield. These positive soil and plant results indicate the positive influences of biofertilizers as a supplementary fertilizer strategy for integrating soil application with NPK fertilizers, allocating a portion of mineral fertilizers for foliar spraying for sustainable agriculture using virgin sandy soils that can be used for the expansion of crop cultivation.

Author Contributions: Conceptualization, M.A.A. and M.M.R.; data curation, H.E.E.B., E.E.B., R.A.Q., H.M.A.-Y. and E.F.A.; formal analysis, M.A.A., M.M.R., H.E.E.B., E.E.B., R.A.-Q., H.M.A.-Y. and E.F.A.; investigation, M.A.A., M.M.R., H.E.E.B., E.E.B., R.A.-Q., H.M.A.-Y. and E.F.A.; methodology, M.A.A., M.M.R., H.E.E.B., E.E.B., R.A.-Q., H.M.A.-Y. and E.F.A.; resources, M.A.A., M.M.R., H.E.E.B., E.E.B., R.A.-Q., H.M.A.-Y. and E.F.A.; software, M.A.A., H.E.E.B., E.E.B., R.A.-Q. and H.M.A.-Y.; writing—original draft, M.A.A., H.E.E.B., E.E.B. and H.M.A.-Y.; writing—review and editing, M.A.A., M.M.R. and E.F.A. All authors have read and agreed to the published version of the manuscript.

Funding: The Deanship of Scientific Research at Taif University through the research number TURSP2020/199 is acknowledged.

Institutional Review Board Statement: Not applicable.

Informed Consent Statement: Not applicable. 
Data Availability Statement: The data presented in this study are available upon request from the corresponding author.

Acknowledgments: The authors are thankful to the Taif University Researchers Supporting Project number (TURSP-2020/199), Taif University, Taif, Saudi Arabia for providing the financial support and research facilities. The authors also greatly acknowledge and thank Colin Pain, MED Soil Research Group and Professor of Soil Science at Seville University, Spain for reviewing the draft manuscript and providing constructive comments, and Abd El-Aty M. Ibrahim, Fayum University for helping with the production of figures.

Conflicts of Interest: The authors declare no conflict of interest.

\section{References}

1. Alshankiti, A.; Gill, S. Integrated Plant Nutrient Management for Sandy Soil Using Chemical Fertilizers, Compost, Biochar and Biofertilizers-Case Study in UAE. J. Arid Land Stud. 2016, 26, 101-106.

2. Pain, C.F.; Abdelfattah, M.A. Landform evolution in the arid northern United Arab Emirates: Impacts of tectonics, sea level changes and climate. Catena 2015, 134, 14-29. [CrossRef]

3. Al-Muaini, A.H.; Green, S.R.; Abou Dahr, W.A.; Al-Yamani, W.; Abdelfattah, M.A.; Pangilinan, R.; McCann, I.; Dakheel, A.; Abdullah, A.; Kennedy, L.; et al. Sustainable Irrigation of Date Palms in the Hyper-Arid United Arab Emirates: A Review. Chron. Hortic. 2019, 4, 30-36.

4. Ksiksi, T.S.; Trueman, R.; Abdelfattah, M.A.; Mousa, M.T.; Almarzouqi, A.Y.; Barahim, S.A. Above and belowground carbon pools are affected by dominant floral species in hyper-arid environments. F1000Research 2019, 8, 1043. [CrossRef]

5. Abdelfattah, M.A. Pedogenesis, land management and soil classification in hyper-arid environments: Results and implications from a case study in the United Arab Emirates. Soil Use Manag. J. 2013, 29, 279-294. [CrossRef]

6. Idowu, O.A.; Lorentz, S.A.; Annandale, J.G.; McCartney, M.P.; Jovanovic, N.Z. Assessment of the Impact of Irrigation with Low-quality Mine Water on Virgin and Rehabilitated Soils in the Upper Olifants Basin. Mine Water Environ. 2008, $27,2-11$. [CrossRef]

7. Wilson, I.G. Ergs. Sediment. Geol. 1973, 10, 77-106. [CrossRef]

8. Fryberger, S.G. Dune forms and wind regime. In A Study of Global Sand Seas, U.S. Geological Survey Professional Paper 1052; McKee, E.D., Ed.; US Government Printing Office: Washington, DC, USA, 1979; pp. 137-170.

9. Thomas, D.S.G. Arid Zone Geomorphology: Process, Forms and Change in Drylands, 2nd ed.; John Wiley \& Sons Ltd.: Chichester, UK, 1997; p. 713.

10. Pye, K.; Tsoar, H. Aeolian Sand and Sand Dunes; Unwin Hyman: London, UK, 1990; p. 396.

11. Bubenzer, O.; Embabi, N.S.; Ashour, M.M. Sand Seas and Dune Fields of Egypt. Geosci 2020, 10, 101. [CrossRef]

12. Brataševec, K.; Sivilotti, P.; Vodopivec, B.M. Soil and foliar fertilization affects mineral contents in Vitis vinifera L. cv. 'rebula' leaves. J. Soil Sci. Plant Nutr. 2013, 13, 650-663.

13. Fregoni, M. Viticoltura di Qualità; Stampa Grafiche Lama: Piacenza, Italy, 1998; pp. 597-680.

14. Kannan, S. Foliar Fertilization for Sustainable Crop Production. In Genetic Engineering, Biofertilization, Soil Quality and Organic Farming; Lichtfouse, E., Ed.; Sustainable Agriculture Reviews 4; Springer: Berlin/Heidelberg, Germany, 2010 ; pp. $371-402$.

15. Tejada, M.; Gonzalez, J.L. Effects off foliar application of a byproduct of the two-step olive oil mill process on rice yield. Europ. J. Agron. 2004, 21, 31-40. [CrossRef]

16. Kaya, C.; Higgs, D. Response of tomato (Lycopersicon esculentum L.) cultivars to foliar application of zinc when grown in sand culture at low zinc. Sci. Hortic. 2002, 93, 53-64. [CrossRef]

17. Jamal, Z.; Hamayun, M.; Ahmed, N.; Chaudhary, M.F. Effect of soil and foliar application of different concentrations of NPK and foliar application of $\left(\mathrm{NH}_{4}\right)_{2} \mathrm{SO}_{4}$ on different yield parameters in wheat. Asian J. Agron. 2006, 5, 251-256.

18. Dewdar, M.D.H.; Rady, M.M. Influence of soil and foliar applications of potassium fertilization on growth, yield and fiber quality traits in two Gossypium barbadense L. varieties. Afr. J. Agric. Res. 2013, 8, 2211-2215.

19. Basavaraj, P.; Chetan, H.T. Folair fertilization of nutrients. Marumegh 2018, 3, 49-53.

20. Rady, M.M. Effects on growth, yield, and fruit quality in tomato (Lycopersicon esculentum Mill.) using a mixture of potassium humate and farmyard manure as an alternative to mineral-N fertiliser. J. Hortic. Sci. Biotechnol. 2011, 86, $249-254$.

21. Rady, M.M.; Semida, W.M.; Hemida, K.A.; Abdelhamid, M.T. The effect of compost on growth and yield of Phaseolus vulgaris plants grown under saline soil. Int. J. Recycl. Org. Waste Agric. 2016, 5, 311-321. [CrossRef]

22. Alharby, H.F.; Al-Zahrani, H.S.; Hakeem, K.R.; Alsamadany, H.; Desoky, E.-S.M.; Rady, M.M. Silymarin-Enriched Biostimulant Foliar Application Minimizes the Toxicity of Cadmium in Maize by Suppressing Oxidative Stress and Elevating Antioxidant Gene Expression. Biomolecules 2021, 11, 465. [CrossRef]

23. Kusparwanti, T.R.; Eliyatiningsih; Wardana, R. Application Legume Compost with Bio-Activator Trichoderma sp as Inorganic Fertilizer Substitution in Sweet Corn (Zea mays L. Saccharata) Cultivation. IOP Conf. Series. Earth Environ. Sci. 2020, 411, 012063. [CrossRef] 
24. Bamagoos, A.A.; Alharby, H.F.; Belal, E.E.; Khalaf, A.E.A.; Abdelfattah, M.A.; Rady, M.M.; Ali, E.F.; Mersal, G.A.M. PhosphateSolubilizing Bacteria as a Panacea to Alleviate Stress Effects of High Soil $\mathrm{CaCO}_{3}$ Content in Phaseolus vulgaris with Special Reference to P-Releasing Enzymes. Sustainability 2021, 13, 7063. [CrossRef]

25. Giller, K.E.; McDonagh, J.F.; Cadisch, G. Can biological nitrogen fixation sustain agriculture in the tropics? In Soil Science and Sustainable Land Management in the Tropics; Syers, P.K., Rimmer, D.L., Eds.; C.A.B International: Wallingford, UK, 1994; pp. 173-191

26. Kumwenda, J.D.T.; Waddington, S.R.; Snapp, S.S.; Jones, R.B.; Blackie, M.J. Soil Fertility Managementfor Smallholder Maize-Based Cropping Systems of Southern Afnca: A Review. Network Working Paper No. 1. Soil Fertility Network for Maize-Based Cropping Systems in Countries of Southern Africa; CIMMYT: Harare, Zimbabwe, 1995; p. 34.

27. Cayuela, M.L.; Mondini, C.; Insam, H.; Sinicco, T.; Whittle, F.I. Plant and animal wastes composting: Effects of the N source on process performance. Bioresour. Technol. 2009, 100, 3097-3106. [CrossRef]

28. Buttery, B.R.; Park, S.l.; Hume, D.F.J. Potential for increasing nitrogen fixation in grain legumes. J. Plant Sci. 1992, 72, 323-349. [CrossRef]

29. Senjobi, B.A. Comparative Assessment of the Effect of Land Use and Land Type on Soil Degradation and Agricultural Productivity in Ogun State, Nigeria. Ph.D. Thesis, Department of Agronomy, University of Ibadan, Ibadan, Nigeria, 2007, unpublished.

30. Page, A.I.; Miller, R.H.; Keeny, D.R. Methods of soil analysis. In Part II. Chemical and Microbiological Methods, 2nd ed.; Amer Soc Agron: Madison, WI, USA, 1982; pp. 225-246.

31. Klute, A.; Dirksen, C. Hydraulic conductivity and diffusivity. Laboratory methods. Methods of Soil Analysis-Part 1. Phys. Mineral. Methods 1986, 9, 687-734.

32. OECD. "Common Bean (Phaseolus Vulgaris)", in Safety Assessment of Transgenic Organisms in the Environment, Volume 6: OECD Consensus Documents; OECD Publishing: Paris, France, 2016. [CrossRef]

33. Carter, M.R.; Gregorich, E.G. Soil Sampling and Methods of Analysis, 2nd ed.; Taylor \& Francis Group: Abingdon, UK, 2006.

34. Hope, C.F.A.; Burns, R.G. Activity, origins and location of cellulases in a silt loam soil. Biol. Fertil. Soils 1987, 5, 164-170. [CrossRef]

35. Schinner, F.; von Mersi, W. Xylanase-, CM-cellulase- and invertase activity in soil: An improved method. Soil Biol. Biochem. 1990, 22, 511-515. [CrossRef]

36. Miller, G.L. Use of dinitrosalicylic acid reagent for the determination of glucose. Anal. Chem. 1972, 31, 426-428. [CrossRef]

37. Kandeler, E.; Gerber, H. Short-term assay of soil urease activity using colorimetric determination of ammonium. Biol. Fertil. Soils 1988, 6, 68-72. [CrossRef]

38. Johnson, J.L.; Temple, K.L. Some variables affecting the measurement of "catalase activity" in soil. Soil Sci. Soc. Am. J. 1964, 28, 207-209. [CrossRef]

39. Stepniewska, Z.; Wolinska, A.; Ziomek, J. Response of soil catalase activity to chromium contamination. J. Environ. Sci. 2009, 21, 1142-1147. [CrossRef]

40. King, E.J. Micro-Analysis in Medical Biochemistry; J. \& A. Churchill: London, UK, 1951.

41. Olsen, S.R. Estimation of Available Phosphorus in Soils by Extraction with Sodium Bicarbonate; US Department of Agriculture: Colorado, CO, USA, 1954.

42. Chapman, H.D. Cation-Exchange capacity. In Methods of Soil Analysis: Part 2 Chemical and Microbiological Properties; Norman, A.G., Ed.; Madison, WI, USA, 1965; Volume 9, pp. 891-901. Available online: https:/ / doi.org/10.2134/agronmonogr9.2.c6 (accessed on 16 June 2021).

43. Arnon, D.I. Copper enzymes in isolated chloroplasts, polyphenoxidase in Beta vulgaris. Plant Physiol. 1949, 24, 1-15. [CrossRef]

44. Li, P.M.; Cai, R.G.; Gao, H.Y.; Peng, T.; Wang, Z.L. Partitioning of excitation energy in two wheat cultivars with different grain protein contents grown under three nitrogen applications in the field. Physiol. Plant. 2007, 129, 822-829. [CrossRef]

45. Maxwell, K.; Johnson, G.N. Chlorophyll fluorescence-A practical guide. J. Exp. Bot. 2000, 51, 659-668. [CrossRef] [PubMed]

46. Clark, A.J.; Landolt, W.; Bucher, J.B.; Strasser, R.J. Beech (Fagus sylvatica) response to ozone exposure assessed with a chlorophyll a fluorescence performance index. Environ. Pollut. 2000, 109, 501-507. [CrossRef]

47. Osman, A.S.; Rady, M.M. Ameliorative effects of sulphur and humic acid on the growth, antioxidant levels, and yields of pea (Pisum sativum L.) plants grown in reclaimed saline soil. J. Hortic. Sci. Biotechnol. 2014, 87, 626-632. [CrossRef]

48. Irigoyen, J.J.; Emerich, D.W.; Sanchez-Diaz, M. Water stress induced changes in the concentrations of proline and total soluble sugars in nodulated alfalfa (Medicago sativa) plants. Plant Physiol. 1992, 8, 455-460. [CrossRef]

49. Bates, L.S.; Waldren, R.P.; Teare, I.D. Rapid determination of free proline for water stress studies. Plant Soil 1973, 39, $205-207$. [CrossRef]

50. Griffth, O.W. Determination of glutathione and glutathione disulfide using glutathione reductase and 2 vinyl pyridine. Anal. Biochem. 1980, 106, 207-212. [CrossRef]

51. Mukherjee, S.P.; Choudhari, M.A. Implications of water stress induced changes in the levels of endogenous ascorbic acid and hydrogen peroxide in Vigna seedlings. Physiol. Plant. 1983, 58, 116-170. [CrossRef]

52. Giannopolitis, C.N.; Ries, S.K. Superoxide dismutases. I. Occurrence in higher plants. Plant Physiol. 1977, 59, 309-314. [CrossRef]

53. Aebi, H. Catalase in vitro. Methods Enzymol. 1984, 105, 121-126.

54. Putter, J. Peroxidase. In Methods of Enzymatic Analysis; Bergmeyer, H.U., Ed.; Verlag Chemie: Weinhan, MA, USA, 1974; pp. 685-690. 
55. Bradford, M.M. A rapid and sensitive for the quantitation of microgram quantities of protein utilizing the principle of protein-dye binding. Anal. Biochem. 1976, 72, 248-254. [CrossRef]

56. Shapiro, S.S.; Wilk, M.B. An Analysis of Variance Test for Normality (Complete Samples). Biometrika 1965, 52, 591-611. [CrossRef]

57. Razali, N.; Wahi, Y.B. Power comparisons of Shapiro-Wilk, Kolmogorov-Smirnov, Lilliefors and Anderson-Darling tests. J. Stat. Model. Anal. 2011, 2, 21-33.

58. Malhi, G.S.; Kaur, M.; Kaushik, P. Impact of climate change on agriculture and its mitigation strategies: A review. Sustainability 2021, 13, 1318. [CrossRef]

59. Abdelfattah, M.A. Climate Change Impacts on Water Resources and Food Security in Egypt and Possible Adaptive Measures-A review. In Climate Change, Food Security, and Sustainable Smart Agriculture; Behnassi, M., Mirza, B.B., El Haiba, M., Reed, M., Eds.; Springer Science + Business Media, B.V.: Berlin, Germany, 2021; in press.

60. Shahid, S.A.; Abdelfattah, M.A.; Taha, F.K. Developments in Soil Salinity Assessment and Reclamation, Innovative Thinking and Use of Marginal Soil and Water Resources in Irrigated Agriculture; Springer Science + Business Media, B.V.: Berlin, Germany, 2013 ; p. 808. ISBN 978-94-007-5684-7.

61. Shahid, S.A.; Taha, F.K.; Abdelfattah, M.A. Developments in Soil Classification, Land Use Planning and Policy Implications, Innovative Thinking of Soil Inventory for Land Use Planning and Management of Land Resources; Springer Science + Business Media, B.V.: Berlin, Germany, 2013; p. 858. ISBN 978-94-007-5331-0.

62. Shahid, S.A.; Abdelfattah, M.A.; Wilson, M.A.; Kelley, J.A.; Chiaretti, J.V. United Arab Emirates Keys to Soil Taxonomy; Springer Science + Business Media, B.V.: Berlin, Germany, 2014; ISBN 978-94-007-7419-3.

63. Pain, C.F.; Abdelfattah, M.A.; Shahid, S.A.; Ditzler, C. Soil-landform relationships in the arid Northern United Arab Emirates. In Geopedology, An Integration of Geomorphology and Pedology for Soil and Landscape Studies; Zinck, J.A., Metternicht, G., Bocco, G., Del Valle, H.F., Eds.; Springer Science + Business Media, B.V.: Berlin, Germany, 2016; pp. 211-225. [CrossRef]

64. Shahid, S.A.; Abdelfattah, M.A.; Mahmoudi, H. Innovations in soil chemical analyses-New ECe and total ions relationship in Abu Dhabi Emirate soils. In Developments in Soil Classification, Land Use Planning and Policy Implications, Innovative Thinking of Soil Inventory for Land Use Planning and Management of Land Resources; Shahid, S.A., Taha, F.K., Abdelfattah, M.A., Eds.; Springer Science + Business Media, B.V.: Berlin, Germany, 2013; pp. 799-812. ISBN 978-94-007-5331-0.

65. Shahid, S.A.; Abdelfattah, M.A.; Othman, Y.; Kumar, A.; Taha, F.K.; Kelley, J.A.; Wilson, M.A. Innovative Thinking for Sustainable Use of Terrestrial Resources in Abu Dhabi Emirate Through Scientific Soil Inventory and Policy Development. In Developments in Soil Classification, Land Use Planning and Policy Implications, Innovative Thinking of Soil Inventory for Land Use Planning and Management of Land Resources; Shahid, S.A., Taha, F.K., Abdelfattah, M.A., Eds.; Springer Science + Business Media, B.V.: Berlin, Germany, 2013; pp. 3-49. ISBN 978-94-007-5331-0.

66. Shendi, M.M.; Abdelfattah, M.A.; Harbi, A. Spatial monitoring of soil salinity and prospective conservation study for Sinnuris District soils, Fayoum, Egypt. In Developments in Soil Salinity Assessment and Reclamation, Innovative Thinking and Use of Marginal Soil and Water Resources in Irrigated Agriculture; Shahid, S.A., Abdelfattah, M.A., Taha, F.K., Eds.; Springer Science + Business Media, B.V.: Berlin, Germany, 2013; pp. 199-217. ISBN 978-94-007-5684-7.

67. Kelley, J.A.; Wilson, M.A.; Abdelfattah, M.A.; Shahid, S.A. Quality Assurance Standards: USDA Perspective of the Extensive Soil Survey of Abu Dhabi Emirate. In Developments in Soil Classification, Land Use Planning and Policy Implications, Innovative Thinking of Soil Inventory for Land Use Planning and Management of Land Resources; Shahid, S.A., Taha, F.K., Abdelfattah, M.A., Eds.; Springer Science + Business Media, B.V.: Berlin, Germany, 2013; pp. 813-822. ISBN 978-94-007-5331-0.

68. King, P.; Grealish, G.; Shahid, S.A.; Abdelfattah, M.A. Land evaluation interpretations-Soil Survey of Abu Dhabi Emirate. In Developments in Soil Classification, Land Use Planning and Policy Implications, Innovative Thinking of Soil Inventory for Land Use Planning and Management of Land Resources; Shahid, S.A., Taha, F.K., Abdelfattah, M.A., Eds.; Springer Science + Business Media, B.V.: Berlin, Germany, 2013; pp. 147-164. ISBN 978-94-007-5331-0.

69. Wilson, M.A.; Shahid, S.A.; Abdelfattah, M.A.; Kelley, J.A.; Thomas, J.E. Anhydrite formation on the coastal sabkha of Abu Dhabi, United Arab Emirates. In Developments in Soil Classification, Land Use Planning and Policy Implications, Innovative Thinking of Soil Inventory for Land Use Planning and Management of Land Resources; Shahid, S.A., Taha, F.K., Abdelfattah, M.A., Eds.; Springer Science + Business Media, B.V.: Berlin, Germany, 2013; pp. 175-202. ISBN 978-94-007-5331-0.

70. Verboom, W.H.; Pate, J.S.; Abdelfattah, M.A.; Shahid, S.A. Effects of plants on soil forming processes: Case studies from arid environments. In Developments in Soil Classification, Land Use Planning and Policy Implications, Innovative Thinking of Soil Inventory for Land Use Planning and Management of Land Resources; Shahid, S.A., Taha, F.K., Abdelfattah, M.A., Eds.; Springer Science + Business Media, B.V.: Berlin, Germany, 2013; pp. 329-344. ISBN 978-94-007-5331-0.

71. Clark, S. Organic farming and climate change: The need for innovation: An opinion. Sustainability 2020, 12, 7012. [CrossRef]

72. Gransee, A.; Fuhrs, H. Magnesium mobility in soils as a challenge for soil and plant analysis magnesium fertilization and root uptake under adverse growth conditions. Plant Soil 2013, 368, 5-21. [CrossRef]

73. Rehman, H.; Alharby, H.F.; Alzahrani, Y.; Rady, M.M. Magnesium and organic biostimulant integrative application induces physiological and biochemical changes in sunflower plants and its harvested progeny on sandy soil. Plant Physiol. Biochem. 2018, 126, 97-105. [CrossRef] 
74. Shahid, S.A.; Taha, F.K.; Ismail, S.; Al Dakheel, A.; Abdelfattah, M.A. Turning Adversity into an Advantage for Food Security Through Improving Soil Quality and Providing Production Systems for Marginal Saline Lands: ICBA Perspectives and Approaches. In Sustainable Agriculture Development, Recent Approaches in Resources Management and Environmentally-Balanced Production Enhancement; Behnassi, M., Shahid, S.A., D'Silva, J., Eds.; Springer Science + Business Media B.V.: Berlin, Germany, 2011.

75. Buragohain, S.; Sarma, B.; Nath, D.J.; Gogoi, N.; Meena, R.S.; Lal, R. Effect of 10 years of biofertiliser use on soil quality and rice yield on an Inceptisol in Assam, India. Soil Res. 2018, 56, 49-58. [CrossRef]

76. Belal, E.E.; El Sowfy, D.M.; Rady, M.M. Integrative soil application of humic acid and sulfur improves saline calcareous soil properties and barley plant performance. Commun. Soil Sci. Plant Anal. 2019, 50, 1919-1930. [CrossRef]

77. Manirakiza, N.; Şeker, C. Effects of compost and biochar amendments on soil fertility and crop growth in a calcareous soil. J. Plant Nutr. 2020, 43, 3002-3019. [CrossRef]

78. Rady, M.M.; El-Shewy, A.A.; Seif El-Yazal, M.A.; Abd El-Gawwad, I.F.M. Integrative application of soil P-solubilizing bacteria and foliar nano $\mathrm{p}$ improves Phaseolus vulgaris plant performance and antioxidative defense system components under calcareous soil conditions. J. Soil Sci. Plant Nutr. 2020, 20, 820-839. [CrossRef]

79. Sinha, S.K.; Kumar, V.; Jha, C.K. Effect of Integrated use of Bio-Compost and Nitrogen on Productivity and Soil Properties of Sugarcane Plant-Ratoon System in Calcareous Soil. SugarTech 2017, 19, 485-491. [CrossRef]

80. Wu, S.C.; Cao, Z.H.; Li, Z.G.; Cheung, K.C.; Wong, M.H. Effects of biofertilizer containing N-fixer, P and K solubilizers and AM fungi on maize growth: A greenhouse trial. Geoderma 2005, 125, 155-166. [CrossRef]

81. Valarini, P.J.; Díaz, M.C.; Gascó, J.M.; Guerrero, F.; Tokeshi, H. Assessment of soil properties by organic matter and EMMicroorganisms incorporation. Rev. Bras. De Ciência Do Solo 2003, 27, 519-525. [CrossRef]

82. Fischer, D.; Glaser, B. Synergisms between compost and biochar for sustainable soil amelioration. In Management of Organic Waste; Kumar, S., Ed.; In Tech.: London, UK, 2012; pp. 168-198. ISBN 978-953-307-925-7.

83. Aboukila, E.F.; Nassar, I.N.; Rashad, M.; Hafez, M.; Norton, J.B. Reclamation of calcareous soil and improvement of squash growth using brewers' spent grain and compost. J. Saudi Soc. Agric. Sci. 2018, 17, 390-397. [CrossRef]

84. Nath, D.J.; Ozha, B.; Barooah, R.C.; Borah, D.K. Effect of integrated nutrient management on soil enzymes, microbial biomass carbon and bacterial populations under rice (Oryza sativa)-wheat (Triticum aestivum) sequence. Indian J. Agric. Sci. 2012, 81, $1143-1148$.

85. Macci, C.; Doni, S.; Peruzzi, E.; Masciandaro, G.; Mennone, C.; Ceccanti, B. Almond tree and organic fertilization for soil quality improvement in southern Italy. J. Environ. Manag. 2010, 95, 215-222. [CrossRef] [PubMed]

86. Swarnalakshmi, K.; Prasanna, R.; Kumar, A.; Pattnaik, S.; Chakravarty, K.; Shivay, Y.S.; Singh, R.; Saxena, A.K. Evaluating the influence of novel cyanobacterial bio filmed biofertilizers on soil fertility and plant nutrition in wheat. Euro. J. Soil Biol. 2013, 55, 107-116. [CrossRef]

87. Hamayun, M.; Khan, S.A.; Khan, A.L.; Shinwari, Z.K.; Ahmad, N.; Kim, Y.H.; Lee, I.J. Effect of foliar and soil application of nitrogen, phosphorus and potassium on yield components of lentil. Pak. J. Bot. 2011, 43, 391-396.

88. Rahman, M.A.; Rahman, M.M.; Begum, M.F.; Alam, M.F. Effect of bio compost, cow dung compost and NPK fertilizers on growth, yield and yield components of chili. Int. J. Biosci. 2012, 2, 51-55.

89. Sandoval, A.P.; Yáñez-Chávez, L.G.; Sánchez-Cohen, I.; Samaniego-Gaxiola, J.A.; Trejo-Calzada, R. Hydrogel, biocompost and its effect on photosynthetic activity and production of forage maize (Zea mays L.) plants. Acta Agron. 2017, 66, 63-68.

90. Sinclair, T.R.; Ludlow, M.M. Influence of soil water supply on the plant water balance of four tropical grain legumes. Aust. J. Plant Physiol. 1986, 13, 329-341. [CrossRef]

91. Nusrat, N.; Shahbaz, M.; Perveen, S. Modulation in growth, photosynthetic efficiency, activity of antioxidants and mineral ions by foliar application of glycinebetaine on pea (Pisum sativum L.) under salt stress. Acta Physiol. Plant. 2014, 36, 2985-2998. [CrossRef]

92. Rady, M.M.; Rehman, H.U. Supplementing organic biostimulants into growing media enhances growth and nutrient uptake of tomato transplants. Sci. Hortic. 2016, 203, 192-198. [CrossRef]

93. Rady, M.M.; Elrys, E.S.; Abo El-Maati, M.E.; Desoky, E.M. Interplaying roles of silicon and proline effectively improve salt and cadmium stress tolerance in Phaseolus vulgaris plant. Plant Physiol. Biochem. 2019, 139, 558-568. [CrossRef] [PubMed]

94. Semida, W.M.; Hemida, K.A.; Rady, M.M. Sequenced ascorbate-proline-glutathione seed treatment elevates cadmium tolerance in cucumber transplants. Ecotoxicol. Environ. Saf. 2018, 154, 171-179. [CrossRef] [PubMed]

95. Alharby, H.F.; Alzahrani, H.S.; Alzahrani, Y.; Alsamadany, H.; Hakeem, K.R.; Rady, M.M. Maize grain extract enriched with polyamines alleviates drought stress in Triticum aestivum through up-regulation of the ascorbate-glutathione cycle, glyoxalase system, and polyamine gene expression. Agronomy 2021, 11, 949. [CrossRef]

96. Rady, M.M.; Desoky, E.-S.M.; Ahmed, S.M.; Majrashi, A.; Ali, E.F.; Arnaout, S.M.A.; Selem, E. Foliar Nourishment with NanoSelenium Dioxide Promotes Physiology, Biochemistry, Antioxidant Defenses, and Salt Tolerance in Phaseolus vulgaris. Plants 2021, 10, 1189. [CrossRef] [PubMed]

97. Rady, M.M.; Boriek, S.H.K.; Abd El-Mageed, T.A.; Seif El-Yazal, M.A.; Ali, E.F.; Hassan, F.A.S.; Abdelkhalik, A. Exogenous Gibberellic Acid or Dilute Bee Honey Boosts Drought Stress Tolerance in Vicia faba by Rebalancing Osmoprotectants, Antioxidants, Nutrients, and Phytohormones. Plants 2021, 10, 748. [CrossRef]

98. Desoky, E.S.; Mansour, E.; Ali, M.M.A.; Yasin, M.A.T.; Abdul-Hamid, M.I.E.; Rady, M.M.; Ali, E.F. Exogenously used 24epibrassinolide promotes drought tolerance in maize hybrids by improving plant and water productivity in an arid environment. Plants 2021, 10, 354. [CrossRef] 
99. Semida, W.M.; Abdelkhalik, A.; Mohamed, G.F.; Abd El-Mageed, T.A.; Abd El-Mageed, S.A.; Rady, M.M.; Ali, E.F. Foliar Application of Zinc Oxide Nanoparticles Promotes Drought Stress Tolerance in Eggplant (Solanum melongena L.). Plants 2021, 10, 421. [CrossRef]

100. Conde, C.; Delrot, S.; Geros, H. Physiological, biochemical and molecular changes occurring during olive development and ripening. J. Plant Physiol. 2008, 165, 1545-1562. [CrossRef] [PubMed] 